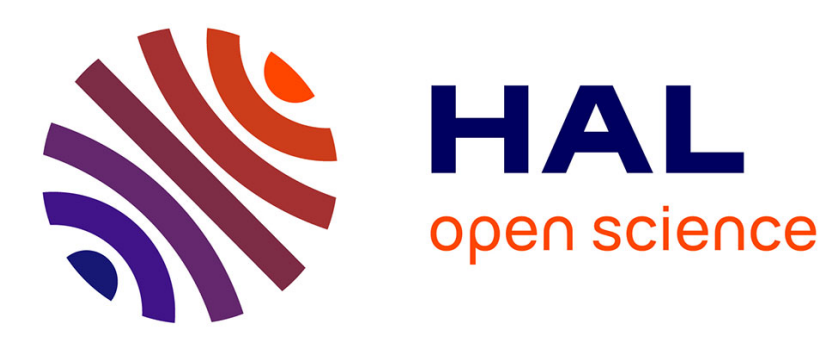

\title{
Stability of equilibrium states in a simple system with unilateral contact and Coulomb friction
}

Stéphanie Basseville, Alain Leger

\section{To cite this version:}

Stéphanie Basseville, Alain Leger. Stability of equilibrium states in a simple system with unilateral contact and Coulomb friction. Archive of Applied Mechanics, 2006, 76 (7), pp.403-428. 10.1007/s00419-006-0040-x . hal-01310671

\section{HAL Id: hal-01310671 \\ https://hal.science/hal-01310671}

Submitted on 3 May 2016

HAL is a multi-disciplinary open access archive for the deposit and dissemination of scientific research documents, whether they are published or not. The documents may come from teaching and research institutions in France or abroad, or from public or private research centers.
L'archive ouverte pluridisciplinaire HAL, est destinée au dépôt et à la diffusion de documents scientifiques de niveau recherche, publiés ou non, émanant des établissements d'enseignement et de recherche français ou étrangers, des laboratoires publics ou privés. 


\title{
Stability of equilibrium states in a simple system with unilateral contact and Coulomb friction
}

\author{
S. Basseville $\cdot$ A. Leger
}

\begin{abstract}
The aim of this paper is to study the stability of equilibrium states in a mechanical system involving unilateral contact with Coulomb friction. Since the assumptions made in classical stability theorems are not satisfied with this class of systems, we return to the basic definitions of stability by studying the time evolution of the distance between a given equilibrium and the solution of a Cauchy problem where the initial conditions are in a neighborhood of the equilibrium. It was recently established that the dynamics is well posed in the case of analytical data. In the present study, we focus in particular on the stability of the equilibrium states under a constant force and deal only with a simple mass-spring system in $\mathbb{R}^{2}$.
\end{abstract}

Keywords Discrete dynamics · Unilateral contact · Coulomb friction · Klarbring's system · Stability

\section{Introduction}

This paper is exactly in line with a previous study [3] in which we explored the equilibrium states of a simple model involving unilateral contact and Coulomb friction. In the latter article, after exploring the set of equilibrium states, we determined their stability by performing a direct numerical study on the dynamics. The criteria used for this purpose were the reliability of the time discretization, which was of the "time stepping" type, and the algorithm used in the "NSCD" software [4]. Since the convergence of this algorithm and its ability to approach the solution of the continuous problem had not yet been established at that time, previous studies dealt mainly with numerical experiments.

The aim of the present study is to provide theoretical proof of the stability of the same simple model.

In [2], we proved the existence of solutions to the dynamic problem with unilateral contact and Coulomb friction, adopting the hypothesis that the external force is integrable using the method described by Monteiro-Marques [6]. After a time discretization, we showed that the "NSCD" algorithm makes it possible to build a sequence of approximations, from which a subsequence converging toward a solution to the continuous problem can be extracted. In [1], it was assumed that the external force was not only integrable but analytical. We proved the uniqueness of the solution in the framework of this hypothesis.

As a consequence of these results, the sequence of approximations given by the algorithm converges uniformly (without extracting a subsequence) toward the unique solution of the problem. This means in particular that the trajectory solution of the continuous problem can be studied starting with estimates based on the discretized problem. After this preliminary step we can study the trajectory starting with any initial data and 
thus analyze the stability of an equilibrium by studying the distance between the equilibrium and a trajectory starting from any point in a neighborhood of the equilibrium in a classical phase space.

\section{Description of the problem}

Let us consider a punctual particle with mass $m$ in $\mathbb{R}^{2}$ located in a quadratic potential well given by a symmetric positive definite stiffness matrix $\underline{\underline{K}}=\left(\begin{array}{cc}K_{\mathrm{N}} & W \\ W & K_{\mathrm{T}}\end{array}\right)$ with positive coefficients. This particle is also subjected to an external force $\underline{F}$. In addition, the particle is constrained to remain in a half-space and the contact with the boundary of this half-space is assumed to hold with Coulomb friction. This system, which has been first given in [5], is represented on Fig. 1. The unilateral contact and Coulomb friction laws are strict, without any regularization. We first recall the statement of the problem, and we give the main theoretical results here without proofs.

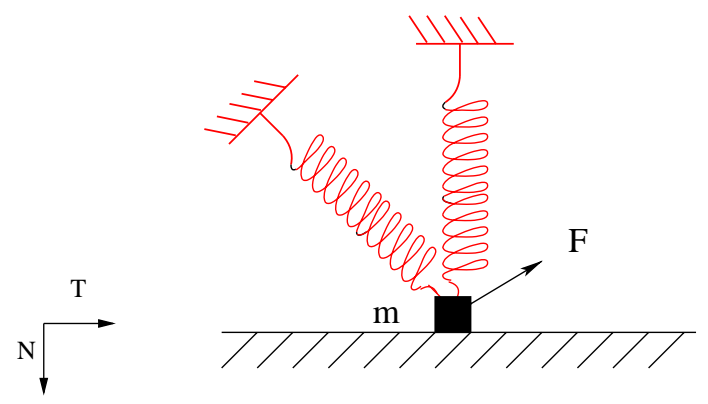

Fig. 1 Klarbring's system

Indices $\mathrm{N}$ and $\mathrm{T}$ denote the normal and tangential components, respectively, of the displacement $\underline{U}$ and the reaction $\underline{R}$. We take MMA $\left([0, \hat{T}] ; \mathbb{R}^{2}\right)$ (motions with measure acceleration) to denote the vector space of the integrable functions of $[0, \hat{T}]$ into $\mathbb{R}^{2}$ whose second derivative in the sense of distributions is a measure. Functions $\underline{U}$ in MMA are continuous and admit left $\underline{U}^{-}$and right $\underline{\dot{U}}^{+}$derivatives in the classical sense, which are functions of bounded variations. We take $\mathcal{M}$ to denote the space of the measures defined on $[0, \hat{T}]$ with values in $\mathbb{R}^{2}$. The evolution problem of mass $m$ reads:

Problem $(\mathcal{P})$ Find $\underline{U} \in \operatorname{MMA}\left([0, \hat{T}] ; \mathbb{R}^{2}\right)$ and $\underline{R} \in \mathcal{M}\left([0, \hat{T}] ; \mathbb{R}^{2}\right)$ such that

$$
\begin{array}{r}
\underline{U}(0)=\underline{U}_{0}, \underline{\dot{U}}^{+}(0)=\underline{V}_{0} \quad \text { (initial condition), } \\
m \underline{\ddot{U}}+\underline{K} \cdot \underline{U}=\underline{F}+\underline{R} \quad \text { (equation of motion), } \\
U_{\mathrm{N}} \leq 0, \quad R_{\mathrm{N}} \leq 0, \quad U_{\mathrm{N}} R_{\mathrm{N}}=0 \quad \text { (unilateral contact) } \\
\forall V \in \mathcal{C}^{0}([0, \hat{T}] ; \mathbb{R}), \quad \int_{[0, \hat{T}]} R_{\mathrm{T}}\left(V-\dot{U}_{\mathrm{T}}^{+}\right)-\mu R_{\mathrm{N}}\left(|V|-\left|\dot{U}_{T}^{+}\right|\right) \geq 0 \quad \text { (Coulomb friction), }
\end{array}
$$

$$
U_{\mathrm{N}}(t)=0 \quad \Longrightarrow \quad \dot{U}_{\mathrm{N}}^{+}(t)=-e \dot{U}_{\mathrm{N}}^{-}(t) \quad \text { (impact law). }
$$

where $\mu$ is the friction coefficient, and the initial condition is assumed to be compatible with the unilateral constraint; $e$ is the restitution coefficient. In the case $e=0$, we can establish that Eqs. (3) and (5) in problem $(\mathcal{P})$ can be replaced by the so-called velocity Signorini conditions:

$$
\begin{aligned}
& U_{\mathrm{N}} \leq 0, \\
& U_{\mathrm{N}}<0 \Longrightarrow R_{\mathrm{N}}=0 \text {, } \\
& U_{\mathrm{N}}=0 \Longrightarrow \dot{U}_{\mathrm{N}}^{+} \leq 0, \quad R_{\mathrm{N}} \leq 0, \quad \dot{U}_{N}^{+} R_{\mathrm{N}}=0 .
\end{aligned}
$$

Comment 2.1 (i) We observe that this formulation has a suitable meaning even for $\underline{R} \in \mathcal{M}\left([0, \hat{T}] ; \mathbb{R}^{2}\right)$. * In particular $\int R_{\mathrm{T}} V$ has a sense for $V$ in $\mathcal{C}^{0}$ (duality).

$\int R_{\mathrm{T}} \dot{U}_{\mathrm{T}}^{+}$has a sense for $\underline{R}$ measure ( $\dot{U}_{\mathrm{T}}^{+}$bounded variation), which would not be so in the case of a usual formulation of Coulomb's law. 
* A velocity with bounded variations is exactly the framework that gives a meaning both to the dynamics in the presence of impact (existence of right and left limits everywhere) and to the initial data.

(ii) This problem has a solution as long as $\underline{F}$ is integrable (this result is obtained by the convergence of a time discretization using a method similar to that given by Monteoro-Marques for $\mathcal{L}^{\infty}$ data [6]); the uniqueness of the trajectory, given the data $\underline{U}_{0}$ and $\underline{V}_{0}$, is obtained only if $\underline{F}$ is an analytical function (counterexample if $\underline{F} \in \mathcal{C}^{\infty}\left([0, \hat{T}] ; \mathbb{R}^{2}\right)$ ).

(iii) The discrete problem that will be used henceforth, where $V$ stands for the discrete values of $\dot{U}$, is written as follows:

\section{Problem $\left(\mathcal{P}_{\mathrm{d}}\right)$}

$$
\left.\begin{array}{c}
h=\frac{\hat{T}}{K}, \\
\underline{V}^{0}=\underline{V}(0), \\
\underline{U}^{0}=\underline{U}(0), \\
\left.\underline{V}^{i+1}=\underline{V}^{i}+\frac{h}{2 m}\left(\underline{F}^{i+1}+\underline{F}^{i}\right)-\underline{\underline{K}}\left(\underline{U}^{i+1}+\underline{U}^{i}\right)\right)+\frac{h}{m} \underline{R}^{i+1}, \\
\underline{U}^{i+1}=\underline{U^{i}}+h \underline{V}^{i+1}, \\
\left|R_{\mathrm{T}}^{i+1}\right| \leq \mu\left|R_{\mathrm{N}}^{i+1}\right|, \\
\text { if }\left|R_{\mathrm{T}}^{i+1}\right|<\mu\left|R_{\mathrm{N}}^{i+1}\right|, \quad \text { then } V_{\mathrm{T}}^{i+1}=0, \\
\text { if }\left|R_{\mathrm{T}}^{i+1}\right|=\mu\left|R_{\mathrm{N}}^{i+1}\right|, \quad \text { such that } R_{\mathrm{T}}^{i+1}=-\lambda V_{\mathrm{T}}^{i+1} .
\end{array}\right\}
$$

* An algorithm of the "time stepping" type is deduced [4], from which we get an iterative dynamical system that will be used to make estimates.

* This algorithm, built from problem $\left(\mathcal{P}_{\mathrm{d}}\right)$, makes heavy use of the equivalence between classical Signorini conditions and velocity Signorini conditions just recalled above. Let us simply note for the moment that the convergence of this algorithm is consequently obtained in the case $e=0$. But the well-posedness of problem $(\mathcal{P})$, that is, the existence and uniqueness of the trajectory for sufficiently smooth data, holds for any $e \in[0,1]$.

The following analysis will be performed in the case $e=0$, and we shall comment on this choice in the conclusion.

\section{The set of equilibrium states}

The equilibrium states are the solutions of problem $\left(\mathcal{P}_{\mathrm{s}}\right)$, which will be referred to here as the static problem, associated with problem $(\mathcal{P})$. Up to the end of this study, the external force $\underline{F}$ will be constant.

Problem $\left(\mathcal{P}_{\mathrm{s}}\right)$ Find $(\underline{U}, \underline{R}) \in\left(\mathbb{R}^{2}, \mathbb{R}^{2}\right)$ such that:

$$
\begin{aligned}
\underline{K} \underline{U} & =\underline{F}+\underline{R}, \\
U_{\mathrm{N}} & \leq 0, \quad R_{\mathrm{N}} \leq 0, \quad U_{\mathrm{N}} R_{\mathrm{N}}=0, \\
\left|R_{\mathrm{T}}\right| & \leq \mu\left|R_{\mathrm{N}}\right|,
\end{aligned}
$$

which gives

$$
\begin{aligned}
& U_{\mathrm{N}}=\frac{A}{\operatorname{det} \underline{\underline{K}}} \text { if } U_{\mathrm{N}}<0, \\
& R_{\mathrm{T}}=\frac{A}{W}+\frac{K_{\mathrm{T}}}{W} R_{\mathrm{N}} \text { if } U_{\mathrm{N}}=0, \text { with } A \equiv K_{\mathrm{T}} F_{\mathrm{N}}-W F_{\mathrm{T}} .
\end{aligned}
$$


The analysis of problem $\mathcal{P}_{\mathrm{s}}$ is described in [3]. Since the system consists of a single particle, we will study the existence of solutions with and without contact separately. Solutions without contact exist only if quantity $A$ is strictly negative. The set of solutions with contact is given in the $\left\{R_{\mathrm{T}}, R_{\mathrm{N}}\right\}$-plane by the intersection between the line $R_{\mathrm{T}}=(A / W)+\left(K_{\mathrm{T}} / W\right) R_{\mathrm{N}}$ and a part of the Coulomb cone, which is possibly delimited by bounds for $R_{\mathrm{N}}$. Figure 2 gives the corresponding solutions in the $\left\{R_{\mathrm{T}}, R_{\mathrm{N}}\right\}$-plane, which have been described in detail in [3]. The thin continuous lines are the boundaries of the Coulomb cone; dashed lines delimit the range of admissible values for $R_{\mathrm{N}}$; on each graph, the thick continuous line is the straight line of equation $R_{\mathrm{T}}=(A / W)+\left(K_{\mathrm{T}} / W\right) R_{\mathrm{N}}$.
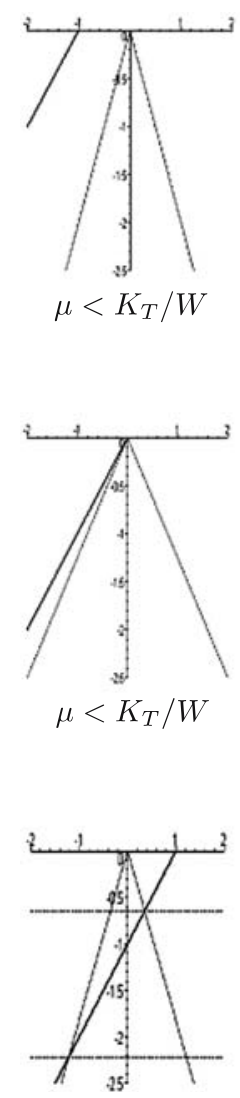

$$
\mu<K_{T} / W
$$

$$
A \equiv K_{T} F_{N}-W F_{T}<0
$$
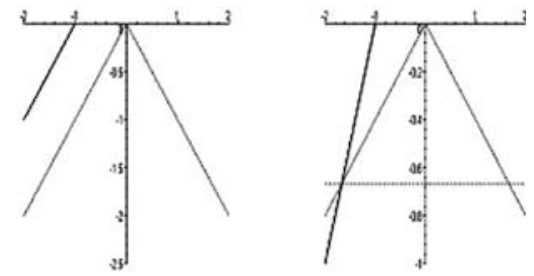

$$
\mu=K_{T} / W
$$$$
\mu>K_{T} / W
$$

$$
A \equiv K_{N} F_{N}-W F_{T}=0
$$
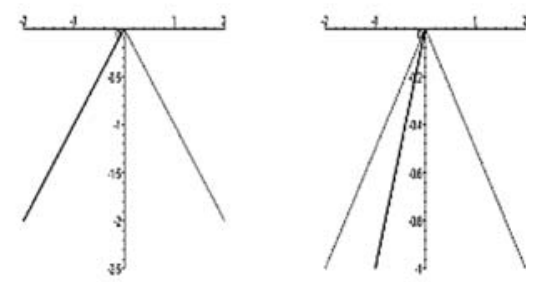

$$
\mu=K_{T} / W
$$$$
\mu>K_{T} / W
$$

$$
A \equiv K_{T} F_{N}-W F_{T}>0
$$
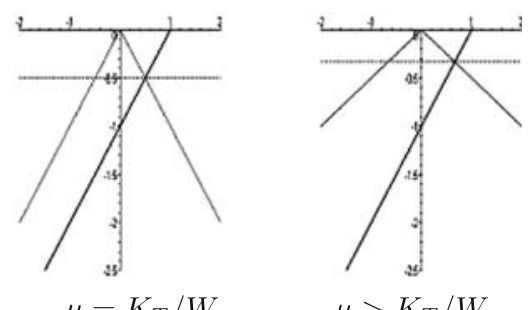

$$
\mu=K_{T} / W
$$

$\mu>K_{T} / W$

Fig. 2 The set of equilibria in the $\left\{R_{T}, R_{N}\right\}$-plane

The dependence of the set of equilibria on the stiffness parameters, friction coefficient, and external loads is summarized in Table 1.

Henceforth we will deal with the stability of these equilibrium states in the case where $\underline{F}=$ Constant. On the one hand, the set of equilibria is completely and explicitly known in this case, and on the other hand, there exists a single analytical trajectory as soon as initial data compatible with the unilateral conditions are given.

\section{Some qualitative phases of the dynamics}

To simplify this description, the proofs will be divided into two mains parts. The first part contains abstract analyses of separate dynamic phases, which will stand for technical lemmas in the second part. The second part itself deals with the stability properties of all the equilibrium states.

In both parts, some partial results will be obtained by performing direct analytical calculations, which will be possible as each of the phases of the motion is smooth, and the successive phases are smoothly matched thanks to the regularity of the solution to problem $(\mathcal{P})$ in the case of a constant force. With other results, 
Table 1 Equilibrium states with respect to parameters $A$ and $\mu$

\begin{tabular}{|c|c|c|c|}
\hline & $\mu<\frac{K_{T}}{W}$ & $\mu=\frac{K_{T}}{W}$ & $\mu>\frac{K_{T}}{W}$ \\
\hline$A<0$ & $\begin{array}{l}1 \text { solution } \\
\text { without contact }\end{array}$ & $\begin{array}{l}1 \text { solution } \\
\text { without contact }\end{array}$ & $\begin{array}{l}1 \text { solution } \\
\text { without contact } \\
+ \\
1 \text { solution } \\
\text { in impending positive slip }\end{array}$ \\
\hline$A=0$ & $\begin{array}{l}1 \text { solution in } \\
\text { grazing contact }\end{array}$ & $\begin{array}{l}1 \text { solution in } \\
\text { grazing contact } \\
+ \\
\text { infinitely many solutions } \\
\text { in impending positive slip }\end{array}$ & $\begin{array}{l}1 \text { solution in } \\
\text { grazing contact } \\
+ \\
\text { infinitely many solutions } \\
\text { in strictly stuck contact }\end{array}$ \\
\hline$A>0$ & $\begin{array}{l}2 \text { solutions in } \\
\text { impending positive and } \\
\text { negative slip } \\
+ \\
\text { infinitely many solutions } \\
\text { in strictly stuck contact }\end{array}$ & $\begin{array}{l}1 \text { solution in impending } \\
\text { negative slip } \\
+ \\
\text { infinitely many solutions } \\
\text { in strictly stuck contact }\end{array}$ & $\begin{array}{l}1 \text { solution in impending } \\
\text { negative slip } \\
+ \\
\text { infinitely many solutions } \\
\text { in strictly stuck contact }\end{array}$ \\
\hline
\end{tabular}

analytical tools of this kind will not be as simple to use, and it will be easier to perform estimates on the iterates of the discrete dynamical system associated with problem $\left(\mathcal{P}_{\mathrm{d}}\right)$ and to conclude with the convergence result.

The whole stability analysis will be performed by perturbing the equilibria obtained with given forces by taking initial data that are out of equilibrium and studying the trajectories without changing the forces. Let us begin by making the following preliminary comment.

Comment 4.1 If we look at the discrete dynamical problem $\left(\mathcal{P}_{\mathrm{d}}\right)$, we observe immediately:

Given an equilibrium in a classical phase space:

(i) A perturbation induced by a displacement is analogous to the second iterate of the discrete dynamics starting from a velocity perturbation. We then ensure that there is no loss of generality by studying only velocity perturbations.

(ii) A perturbation induced by a velocity having nonzero normal and tangential components is analogous to the second iterate of the discrete dynamics starting with a perturbation induced by a velocity where only one of the components differs from zero. Without any loss of generality, we can therefore restrict the analysis to perturbations induced by velocities having only one nonzero component.

Lemma 4.1 Let $\left(\underline{U}^{e q}, \underline{R}^{e q}\right)$ be an equilibrium state with $U_{N}^{e q}=0$ and $V_{0 N}<0$ a perturbation of this equilibrium induced by a normal velocity at $t=0$. The solution of problem $(\mathcal{P})$ will then be such that there exists an impact time $\left.t^{i m p}>0, t^{i m p} \in\right] 0, \hat{T}[$.

Proof After a perturbation $V_{0 \mathrm{~N}}$ compatible with the unilateral conditions, there exists an interval in which the particle moves without contact, since the solution is analytical. During this phase, the evolution is described by the smooth system:

$$
\left\{\begin{array}{l}
m \ddot{U}_{\mathrm{N}}+K_{\mathrm{N}} U_{\mathrm{N}}+W U_{T}=F_{\mathrm{N}} \\
m \ddot{U}_{\mathrm{T}}+W U_{\mathrm{N}}+K_{\mathrm{T}} U_{\mathrm{T}}=F_{\mathrm{T}} \\
U_{\mathrm{N}}(0)=0, U_{\mathrm{T}}(0)=\frac{F_{\mathrm{N}}+R_{\mathrm{N}}^{\mathrm{eq}}}{W} \\
\dot{U}_{\mathrm{N}}(0)=V_{0 N}, \dot{U}_{\mathrm{T}}(0)=0
\end{array} .\right.
$$

The solution is

$$
\underline{U}(t)=\left(a_{1} \cos \left(\alpha_{1} t\right)+b_{1} \sin \left(\alpha_{1} t\right)\right) \underline{\phi}^{1}+\left(a_{2} \cos \left(\alpha_{2} t\right)+b_{2} \sin \left(\alpha_{2} t\right)\right) \underline{\phi}^{2}+\underline{\underline{K}}^{-1} \underline{F},
$$

where $\underline{\underline{K}}^{-1}$ is the inverse of matrix $\underline{\underline{K}}$ and where the constants are given by 


$$
\begin{aligned}
\alpha_{1} & =\sqrt{\frac{\left(K_{\mathrm{N}}+K_{\mathrm{T}}\right)+\sqrt{\left(K_{\mathrm{N}}-K_{\mathrm{T}}\right)^{2}+4 W^{2}}}{2 m}}, \quad \alpha_{2}=\sqrt{\frac{\left(K_{\mathrm{N}}+K_{T}\right)-\sqrt{\left(K_{\mathrm{N}}-K_{\mathrm{T}}\right)^{2}+4 W^{2}}}{2 m}}, \\
\underline{\phi}^{1} & =\left(\frac{K_{\mathrm{N}}-K_{\mathrm{T}}+\sqrt{\left(K_{\mathrm{N}}-K_{\mathrm{T}}\right)^{2}+4 W^{2}}}{2 m}\right), \quad \underline{\phi}^{2}=\left(\frac{1}{\left.\frac{K_{\mathrm{N}}-K_{\mathrm{T}}-\sqrt{\left(K_{\mathrm{N}}-K_{\mathrm{T}}\right)^{2}+4 W^{2}}}{2 m}\right),}\right. \\
a_{1} & =\frac{1}{\phi_{\mathrm{T}}^{1}-\phi_{\mathrm{T}}^{2}}\left\{\frac{A}{\operatorname{det} \underline{\underline{K}}}\left(\phi_{\mathrm{T}}^{2}+\frac{K_{\mathrm{N}}}{W}\right)+\frac{R_{\mathrm{N}}^{e q}}{W}\right\}, \quad a_{2}=-\frac{1}{\phi_{\mathrm{T}}^{1}-\phi_{\mathrm{T}}^{2}}\left\{\frac{A}{\operatorname{det} \underline{\underline{K}}}\left(\phi_{\mathrm{T}}^{1}+\frac{K_{\mathrm{N}}}{W}\right)+\frac{R_{\mathrm{N}}^{e q}}{W}\right\}, \\
b_{1} & =\frac{-V_{0 N} \phi_{\mathrm{T}}^{2}}{\alpha_{1}\left(\phi_{\mathrm{T}}^{1}-\phi_{\mathrm{T}}^{2}\right)}, \quad b_{2}=\frac{V_{0 N} \phi_{\mathrm{T}}^{1}}{\alpha_{2}\left(\phi_{\mathrm{T}}^{1}-\phi_{\mathrm{T}}^{2}\right)}, \\
\underline{\underline{K}} & =\frac{1}{\operatorname{det} \underline{\underline{K}}\left(K_{\mathrm{N}} F_{\mathrm{T}}-W F_{\mathrm{N}}\right) .}
\end{aligned}
$$

If $\left(\alpha_{1} / \alpha_{2}\right)=(p / q) \in \mathbb{Q}$, then $U_{\mathrm{N}}$ is a periodic function. If $\left(\alpha_{1} / \alpha_{2}\right) \notin \mathbb{Q}$, then let $\tilde{U}_{\mathrm{N}}(t)$ be a continuous almost-periodic function of $\mathbb{R}$ into $\mathbb{R}$ that coincides with $U_{\mathrm{N}}(t)$ on $[0, \hat{T}]$. Let $\tau$ be an almost-period of $\tilde{U}_{\mathrm{N}}(t)$. It can then be easily proved that there exists a nonnegative real number $\eta$ such that $\tilde{U}_{\mathrm{N}}(t)$ admits a zero $t^{\text {imp }}$ in $]-\eta+\tau, \eta+\tau\left[\right.$, with $\dot{\tilde{U}}_{N}^{-}\left(t^{\text {imp }}\right)>0$.

The result obtained in [1] shows that $\hat{T}$ can always be taken to be large enough for $\left.t^{\text {imp }} \in\right] 0, \hat{T}[$. Therefore, there will exist an impact time for any $\alpha_{1}$ and $\alpha_{2}$, that is, whatever the constants involved in the problem.

Lemma 4.2 Let $\left(\underline{U}^{e q}, \underline{0}\right)$ be an equilibrium state in grazing contact and $V_{0 T}$ a perturbation of this equilibrium induced by a strictly positive tangential velocity at $t=0$. The solution of problem $(\mathcal{P})$ therefore admits an impact time $t^{i m p}>0, t^{i m p}$ in $] 0, \hat{T}[$.

Proof The equilibrium state is characterized by a displacement $\underline{U}^{e q}=\left(0, F_{\mathrm{N}} / W\right)$ and by a reaction $\underline{R}^{\mathrm{eq}}=0$. This state exists only if $A$ is equal to zero. On the right side of the origin, there exists an interval in which the solution is either always in contact or always without contact. We establish that there exists an interval such that the particle is without contact if the perturbation is a positive tangential velocity at $t=0$.

First, we show that there does not exist any $\eta>0$ such that $U_{\mathrm{N}}(t)=0$ on $[0, \eta]$, since the bilateral positive sliding, which occurs with $R_{\mathrm{N}}(0)=0$, implies $R_{\mathrm{N}}(t)>0$, on an interval at the right of the origin:

$$
\begin{aligned}
& \text { If } \mu<\frac{K_{\mathrm{T}}}{W} \quad\left\{\begin{array}{l}
U_{\mathrm{T}}(t)=V_{0 \mathrm{~T}} \sqrt{\frac{m}{K_{\mathrm{T}}-\mu W}} \sin \left(\sqrt{\frac{K_{\mathrm{T}}-\mu W}{m}} t\right)+\frac{F_{\mathrm{T}}-\mu F_{\mathrm{N}}}{K_{\mathrm{T}}-\mu W}, \\
R_{\mathrm{N}}(t)=W V_{0 \mathrm{~T}} \sqrt{\frac{m}{K_{\mathrm{T}}-\mu W}} \sin \left(\sqrt{\frac{K_{\mathrm{T}}-\mu W}{m}} t\right)>0 \quad \text { with } t \in\left[0, \sqrt{\frac{m}{K_{\mathrm{T}}-\mu W}} \frac{\pi}{2}\right] .
\end{array}\right. \\
& \text { If } \mu=\frac{K_{\mathrm{T}}}{W}\left\{\begin{array}{l}
U_{\mathrm{T}}(t)=V_{\mathrm{T}} 0 \mathrm{~T} t+\frac{F_{\mathrm{N}}}{W}, \\
R_{\mathrm{N}}(t)=W V_{0 \mathrm{~T}} t>0 .
\end{array}\right. \\
& \text { If } \mu>\frac{K_{\mathrm{T}}}{W}\left\{\begin{array}{l}
U_{\mathrm{T}}(t)=\frac{V_{0 \mathrm{~T}}}{2} \sqrt{\frac{m}{K_{\mathrm{T}}-\mu W}}\left(e^{\sqrt{\frac{K_{\mathrm{T}}-\mu W}{m}} t}-e^{-\sqrt{\frac{K_{\mathrm{T}}-\mu W}{m}} t}\right)+\frac{F_{\mathrm{T}}-\mu F_{\mathrm{N}}}{K_{\mathrm{T}}-\mu W}, \\
R_{\mathrm{N}}(t)=\frac{W V_{0 \mathrm{~T}}}{2} \sqrt{\frac{m}{K_{\mathrm{T}}-\mu W}}\left(e^{\sqrt{\frac{K_{\mathrm{T}}-\mu W}{m}} t}-e^{-\sqrt{\frac{K_{\mathrm{T}}-\mu W}{m}} t}\right)>0 .
\end{array}\right.
\end{aligned}
$$

In the case of a perturbation induced by a positive tangential velocity, the evolution of the particule without contact is described by a problem similar to (14) where the initial conditions are:

$$
U_{\mathrm{N}}(0)=0, \quad U_{\mathrm{T}}(0)=\frac{F_{\mathrm{N}}}{W}, \quad \dot{U}_{\mathrm{N}}(0)=0, \quad \dot{U}_{\mathrm{T}}(0)=V_{0 \mathrm{~T}}>0
$$

where the solution is consequently: 


$$
\begin{aligned}
U_{\mathrm{N}}(t) & =\frac{V_{0 \mathrm{~T}}}{\phi_{\mathrm{T}}^{1}-\phi_{\mathrm{T}}^{2}}\left(\frac{\sin \left(\alpha_{1} t\right)}{\alpha_{1}}-\frac{\sin \left(\alpha_{2} t\right)}{\alpha_{2}}\right), \\
U_{\mathrm{T}}(t) & =\frac{V_{0 \mathrm{~T}}}{\phi_{\mathrm{T}}^{1}-\phi_{\mathrm{T}}^{2}}\left(\frac{\sin \left(\alpha_{1} t\right)}{\alpha_{1}} \phi_{\mathrm{T}}^{1}-\frac{\sin \left(\alpha_{2} t\right)}{\alpha_{2}} \phi_{\mathrm{T}}^{2}\right)+\frac{F_{\mathrm{N}}}{W},
\end{aligned}
$$

where $\underline{\phi}_{1}, \underline{\phi}_{2}, \alpha_{1}$, and $\alpha_{2}$ are given in the proof of Lemma 4.1 .

In the neigborhood of $t=0$, the normal displacement $U_{\mathrm{N}}(t)$ is of the form

$$
U_{\mathrm{N}}(t)=\frac{V_{0 \mathrm{~T}}}{\phi_{\mathrm{T}}^{1}-\phi_{\mathrm{T}}^{2}} \frac{\left(\alpha_{2}^{2}-\alpha_{1}^{2}\right)}{6} t^{3}+\mathcal{O}\left(t^{5}\right)
$$

and is strictly negative since $\phi_{\mathrm{T}}^{1}-\phi_{\mathrm{T}}^{2}$ is positive and $\alpha_{2}^{2}-\alpha_{1}^{2}$ is negative. The first relation of (17) then gives the existence of an impact time given by the smallest positive root of this equation.

A similar argument leads to the following corollary.

Corollary 4.1 Let us assume that there exists a time such that the particle is at the vertex of Coulomb's cone with a positive tangential velocity. Then this time is followed by a phase without contact, which is followed in turn by a time of impact $t^{i m p}$.

Lemma 4.3 (1) Assume the data are such that either $A \geq 0$ and $\mu>K_{T} / W$ or $A=0$ for any $\mu$.

Let $V_{0 N}$ be a perturbation of the equilibrium induced by a negative normal velocity at $t=0$.

Let $t^{i m p}>0$ be an impact time such that $\dot{U}_{T}^{-}\left(t^{i m p}\right)>0$. Therefore, there will exist $\eta>0$ such that $\left.\forall V_{0 N} \in\right]-\eta, 0\left[\right.$, and there will exist $\hat{t}>t^{\text {imp }}$ with $\dot{U}_{T}(\hat{t})=0$ and $R_{N}(\hat{t})<0$.

(2) Let $A=0$ with $\mu>K_{T} / W$. Let $V_{0 T}$ be a perturbation of the equilibrium in grazing contact induced by a positive tangential velocity at $t=0$.

Let $t^{i m p}>0$ be an impact time such that $\dot{U}_{T}^{-}\left(t^{i m p}\right)>0$. Therefore, there will exist $\eta>0$ such that $\forall V_{0 T} \in\left[0, \eta\left[\right.\right.$, and there will exist $\hat{t}>t^{i m p}$ with $\dot{U}_{T}(\hat{t})=0$ and $R_{N}(\hat{t})<0$.

Proof We give the proof in the case where $A=0$ and $\mu>\left(K_{\mathrm{T}} / W\right)$. The proof would be similar in the other cases.

Let $\left(\underline{U}^{\mathrm{eq}}, \underline{R}^{\mathrm{eq}}\right.$ ) be an equilibrium of the kind described above and $V_{0 \mathrm{~N}}$ a strictly negative perturbation. The dynamics is the solution to problem $(\mathcal{P})$. According to Lemma 4.1, there exists a time of impact $t^{\text {imp }}$.

After this impact, the evolution will depend on the sign of the tangential velocity $\dot{U}_{\mathrm{T}}\left(V_{0 \mathrm{~N}}, t^{\mathrm{imp}}\left(V_{0 N}\right)\right)=$ $\frac{\mathrm{d} U_{\mathrm{T}}\left(V_{0 \mathrm{~N}}, t^{\mathrm{imp}}\left(V_{0 \mathrm{~N}}\right)\right)}{\mathrm{d} t}$. When the tangential velocity is strictly positive, the particle shows a positive slip. During this phase, the reaction increases. Since the external force is constant, the system will be smooth during the slip and will be governed by an ordinary differential equation, and the evolution of the reaction is given by

$$
\text { if } U_{\mathrm{N}}(t) \equiv 0, \frac{\mathrm{d} R_{\mathrm{N}}(t)}{\mathrm{d} t}=W \dot{U}_{\mathrm{T}}(t)
$$

Since the quantity $W$ is strictly positive, the particle can reach the vertex of the cone and will no longer be in contact (cf. Lemma 4.2). But if the perturbation is sufficiently small, the tangential velocity becomes zero before reaching the vertex of the cone. 


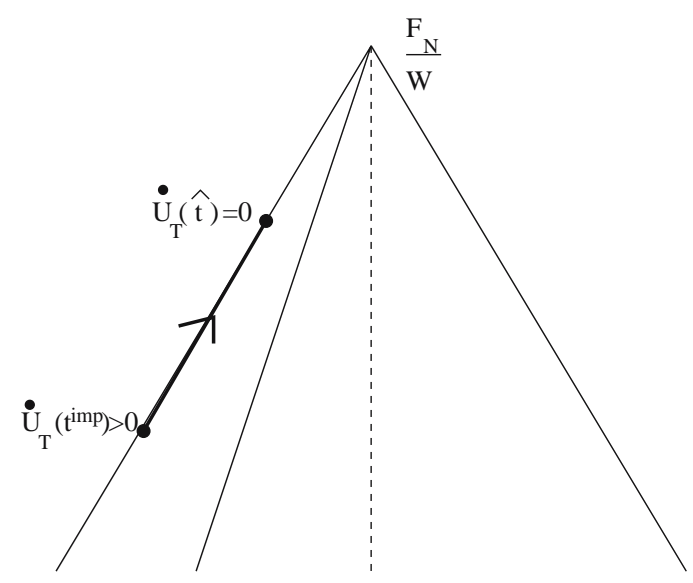

Since the tangential displacement is $U_{\mathrm{T}}=F_{\mathrm{N}} / W$ at the vertex of the cone, this holds if

$$
\exists \hat{t}>t^{\mathrm{imp}} \text { such as } \dot{U}_{\mathrm{T}}(\hat{t})=0 \text { with } U_{\mathrm{T}}(\hat{t})<\frac{F_{\mathrm{N}}}{W} .
$$

Since the tangential velocity is continuous at the impact [1], the positive slip is described by

$$
\left\{\begin{array}{l}
m \ddot{U}_{\mathrm{T}}+\left(K_{\mathrm{T}}-\mu W\right) U_{\mathrm{T}}=F_{\mathrm{T}}-\mu F_{\mathrm{N}} \\
U_{\mathrm{T}}(0)=U_{\mathrm{T}}\left(V_{0 \mathrm{~N}}, t^{\mathrm{imp}}\left(V_{0 \mathrm{~N}}\right)\right) \\
\dot{U}_{\mathrm{T}}(0)=\dot{U}_{\mathrm{T}}^{-}\left(V_{0 \mathrm{~N}}, t^{\operatorname{imp}}\left(V_{0 \mathrm{~N}}\right)\right)
\end{array}\right.
$$

the solution of which is

$$
\begin{aligned}
U_{\mathrm{T}}(t)= & \frac{1}{2}\left\{U _ { \mathrm { T } } \left(V_{0 \mathrm{~N}}, t^{\mathrm{imp}}\left(V_{0 \mathrm{~N}}\right)+\dot{U}_{\mathrm{T}}^{-}\left(V_{0 \mathrm{~N}}, t^{\mathrm{imp}}\left(V_{0 \mathrm{~N}}\right) \sqrt{\frac{m}{\mu W-K_{\mathrm{T}}}}-\frac{F_{\mathrm{T}}-\mu F_{\mathrm{N}}}{K_{\mathrm{T}}-\mu W}\right\} e^{\sqrt{\frac{\mu W-K_{\mathrm{T}}}{m}} t}\right.\right. \\
& +\frac{1}{2}\left\{U _ { \mathrm { T } } \left(V_{0 \mathrm{~N}}, t^{\mathrm{imp}}\left(V_{0 \mathrm{~N}}\right)-\dot{U}_{\mathrm{T}}^{-}\left(V_{0 \mathrm{~N}}, t^{\mathrm{imp}}\left(V_{0 \mathrm{~N}}\right) \sqrt{\frac{m}{\mu W-K_{\mathrm{T}}}}-\frac{F_{\mathrm{T}}-\mu F_{\mathrm{N}}}{K_{\mathrm{T}}-\mu W}\right\} e^{-\sqrt{\frac{\mu W-K_{\mathrm{T}}}{m}} t}\right.\right. \\
& +\frac{F_{\mathrm{T}}-\mu F_{\mathrm{N}}}{K_{\mathrm{T}}-\mu W} .
\end{aligned}
$$

Then condition (19) reads

$$
U_{\mathrm{T}}\left(V_{0 \mathrm{~N}}, t^{\mathrm{imp}}\left(V_{0 \mathrm{~N}}\right)\right)+\dot{U}_{\mathrm{T}}^{-}\left(V_{0 \mathrm{~N}}, t^{\mathrm{imp}}\left(V_{0 \mathrm{~N}}\right)\right) \sqrt{\frac{m}{\mu W-K_{\mathrm{T}}}}-\frac{F_{\mathrm{T}}-\mu F_{\mathrm{N}}}{K_{\mathrm{T}}-\mu W}<0 .
$$

Let us now prove the existence of $\eta>0$ such that condition (22) is satisfied $\left.\forall V_{0 \mathrm{~N}} \in\right]-\eta, 0[$.

We consider the function $V_{0 N}$ defined by the left-hand side of relation (22).

We first assume that the initial perturbation $V_{0 \mathrm{~N}}$ is negative and small enough, $V_{0 \mathrm{~N}}=-\xi_{1}, \xi_{1}>0$.

During the evolution without contact, the normal displacement is

$$
U_{\mathrm{N}}(t)=\frac{R_{\mathrm{N}}^{e q}}{W\left(\phi^{1}-\phi_{\mathrm{T}}^{2}\right)}\left\{\cos \left(\alpha_{1} t\right)-\cos \left(\alpha_{2} t\right)\right\}+\frac{\xi_{1}}{\left(\phi_{\mathrm{T}}^{1}-\phi_{\mathrm{T}}^{2}\right)}\left\{\frac{\sin \left(\alpha_{1} t\right) \phi_{\mathrm{T}}^{2}}{\alpha_{1}}-\frac{\sin \left(\alpha_{2} t\right) \phi_{\mathrm{T}}^{1}}{\alpha_{2}}\right\} .
$$

If $\xi_{1}$ is sufficiently small, then the time of impact will be close to zero. The evolution is given by

$$
U_{\mathrm{N}}(t)=\left\{\frac{R_{\mathrm{N}}^{\mathrm{eq}}}{2 W\left(\phi_{\mathrm{T}}^{1}-\phi_{\mathrm{T}}^{2}\right)}\left(\alpha_{2}^{2}-\alpha_{1}^{2}\right) t-\xi_{1}\right\} t+\mathcal{O}\left(t^{3}\right)
$$


Consequently, the particle is in contact at time

$$
t^{\mathrm{imp}}=\frac{2 W \xi_{1}\left(\phi_{\mathrm{T}}^{1}-\phi_{\mathrm{T}}^{2}\right)}{R_{\mathrm{N}}^{\mathrm{eq}}\left(\alpha_{2}^{2}-\alpha_{1}^{2}\right)} .
$$

At this time, the left-hand side of relation (22) is

$$
\begin{gathered}
x \quad U_{\mathrm{T}}\left(\xi_{1}, t^{\mathrm{imp}}\left(\xi_{1}\right)\right)+\dot{U}_{\mathrm{T}}^{-}\left(\xi_{1}, t^{\mathrm{imp}}\left(\xi_{1}\right)\right) \sqrt{\frac{m}{\mu W-K_{\mathrm{T}}}}-\frac{F_{\mathrm{T}}-\mu F_{\mathrm{N}}}{K_{\mathrm{T}}-\mu W} \\
=-2 \frac{\alpha_{1}^{2} \phi_{\mathrm{T}}^{1}-\alpha_{2}^{2} \phi_{\mathrm{T}}^{2}}{\alpha_{2}^{2}-\alpha_{1}^{2}} \sqrt{\frac{m}{\mu W-K_{\mathrm{T}}}} \xi_{1}+\frac{R_{\mathrm{N}}^{\mathrm{eq}}}{W}+\mathcal{O}\left(\xi_{1}^{2}\right) .
\end{gathered}
$$

$\xi_{1}$ is sufficiently small and quantity

$$
2 \frac{\alpha_{1}^{2} \phi_{\mathrm{T}}^{1}-\alpha_{2}^{2} \phi_{\mathrm{T}}^{2}}{\alpha_{2}^{2}-\alpha_{1}^{2}} \sqrt{\frac{m}{\mu W-K_{\mathrm{T}}}}
$$

is finite. The sign of quantity (23) is that of $R_{\mathrm{N}}^{\mathrm{eq}} / W$, which is strictly negative.

Let us now assume that $V_{0 N}=-\xi_{2}, \xi_{2}>0$ being sufficiently large. The left-hand side of (22) is now

$$
\begin{aligned}
U_{\mathrm{T}}\left(\xi_{2}, t^{\mathrm{imp}}\left(\xi_{2}\right)\right)+\dot{U}_{\mathrm{T}}^{-}\left(\xi_{2}, t^{\mathrm{imp}}\left(\xi_{2}\right)\right) \sqrt{\frac{m}{\mu W-K_{\mathrm{T}}}}-\frac{F_{\mathrm{T}}-\mu F_{N}}{K_{\mathrm{T}}-\mu W} & >U_{\mathrm{T}}\left(\xi_{2}, t^{\mathrm{imp}}\left(\xi_{2}\right)\right)-\frac{F_{\mathrm{T}}-\mu F_{\mathrm{N}}}{K_{\mathrm{T}}-\mu W} \\
& >\frac{R_{\mathrm{N}}^{\mathrm{eq}}}{W}-\frac{\xi_{2} \phi_{\mathrm{T}}^{1} \phi_{\mathrm{T}}^{2}}{\phi_{\mathrm{T}}^{1}-\phi_{\mathrm{T}}^{2}}\left(\frac{1}{\alpha_{1}}-\frac{1}{\alpha_{2}}\right)-\frac{F_{\mathrm{T}}-\mu F_{\mathrm{N}}}{K_{\mathrm{T}}-\mu W},
\end{aligned}
$$

which is positive if

$$
\xi_{2}>\frac{\left(\phi_{\mathrm{T}}^{1}-\phi_{\mathrm{T}}^{2}\right) \alpha_{1} \alpha_{2}}{\left(\alpha_{1}-\alpha_{2}\right) \phi_{\mathrm{T}}^{1} \phi_{\mathrm{T}}^{2}}\left\{\frac{F_{\mathrm{T}}-\mu F_{\mathrm{N}}}{K_{\mathrm{T}}-\mu W}-\frac{R_{\mathrm{N}}^{\mathrm{eq}}}{W}\right\} .
$$

Since the right-hand side of (22) is a continuous function, there exists $\eta \in] \xi_{1}, \xi_{2}$ [ such that condition (22) is satisfied for all $\left.V_{0 \mathrm{~N}} \in\right]-\eta, 0[$.

The proof is the same for the other cases $\left(\mu<\left(K_{\mathrm{T}} / W\right), \quad \mu=\left(K_{\mathrm{T}} / W\right), \quad \mu>\left(K_{\mathrm{T}} / W\right)\right.$, and $A \geq 0$ or $\mu>\frac{K_{T}}{W}$ and $\left.A=0\right)$.

In the same way, we can establish the following lemma 4.4:

Lemma 4.4 (1) Let $A \geq 0$, with $\mu>K_{T} / W$ if $A=0$, and for any $\mu$ if $A>0$.

Let $V_{0 T}$ be a perturbation of the equilibrium induced by a tangential velocity at $t=0$.

Therefore, there exists $\eta>0$ such that $\forall V_{0 T}<\eta$, and there exists $\hat{t}>0$ such that $\dot{U}_{T}(\hat{t})=0$ with $R_{N}(\hat{t})<0$.

(2) Let $A=0$ with $\mu=K_{T} / W$.

Let $V_{0 T}$ be a perturbation of the equilibrium in grazing contact induced by a negative velocity at $t=0$.

Therefore, there exists $\hat{t}>0$ such that $\dot{U}_{T}(\hat{t})=0$ with $R_{N}(\hat{t})<0$.

Lemma 4.5 (1) Let $A \geq 0$, with $\mu>K_{T} / W$ if $A=0$, or any $\mu$ if $A>0$.

Under the assumptions adopted in Lemma 4.4, item 1, there exists $\hat{t}>0$ such that $\dot{U}_{T}(\hat{t})=0$ with $U_{N}(\hat{t})=0$.

If in addition there exists an equilibrium $\left(\underline{\tilde{U}}^{e q}, \underline{\tilde{R}}^{e q}\right)$ such that $R_{N}(\hat{t})=\tilde{R}_{N}^{e q}$, then $\dot{U}_{T}(t) \equiv 0$ and $\dot{U}_{N}(t) \equiv 0 \forall t>\hat{t}$.

(2) Let $A=0$ with $\mu=K_{T} / W$. Under the assumptions adopted in Lemma 4.4, item 2, there exists $\hat{t}>0$ such that $\dot{U}_{T}(\hat{t})=0$ with $U_{N}(\hat{t})=0$. Then, $\dot{U}_{T}(t) \equiv 0$ and $\dot{U}_{N}(t) \equiv 0, \forall t>\hat{t}$. 
Estimate of displacement $\tilde{U}_{\mathrm{N}}^{i+}$

if $\tilde{U}_{\mathrm{N}}^{i+1}<0$, the particle will evolve without contact and the solution will be

$$
\left\{\begin{array}{l}
\left(V_{\mathrm{N}}^{i+1}, V_{\mathrm{T}}^{i+1}\right)=\left(\left(V_{\mathrm{N}}^{i+1}\right)^{\text {free }},\left(V_{\mathrm{T}}^{i+1}\right)^{\text {free }}\right), \\
\left(R_{\mathrm{N}}^{i+1}, R_{\mathrm{T}}^{i+1}\right)=(0,0)
\end{array}\right.
$$

else

if $\left(V_{\mathrm{N}}^{i+1}\right)^{\text {free }}<0$, the particle will ascend and the solution will be

$$
\left\{\begin{array}{l}
\left(V_{\mathrm{N}}^{i+1}, V_{\mathrm{T}}^{i+1}\right)=\left(\left(V_{\mathrm{N}}^{i+1}\right)^{\text {free }},\left(V_{\mathrm{T}}^{i+1}\right)^{\text {free }}\right), \\
\left(R_{\mathrm{N}}^{i+1}, R_{\mathrm{T}}^{i+1}\right)=(0,0)
\end{array}\right.
$$

else

if $\tilde{F}_{\mathrm{T}}^{i+1}-\mu \tilde{F}_{\mathrm{N}}^{i+1} \geq 0$, the particle undergoes positive slip process and the solution will be

$$
\left\{\begin{array}{l}
\left(V_{\mathrm{N}}^{i+1}, V_{\mathrm{T}}^{i+1}\right)=\left(0, \frac{\tilde{F}_{\mathrm{T}}^{i+1}-\mu \tilde{F}_{\mathrm{N}}^{i+1}}{\tilde{M}_{2,2}-\mu \tilde{M}_{1,2}}\right) \\
\left(R_{\mathrm{N}}^{i+1}, R_{\mathrm{T}}^{i+1}\right)=\left(\frac{\tilde{M}_{1,2} V_{\mathrm{T}}^{i+1}-\tilde{F}_{\mathrm{N}}^{i+1}}{h}, \mu R_{\mathrm{N}}^{i+1}\right)
\end{array}\right.
$$

else

if $\tilde{F}_{\mathrm{T}}^{i+1}+\mu \tilde{F}_{\mathrm{N}}^{i+1} \leq 0$, the particle undergoes negative slip process and the solution will be

$$
\left\{\begin{array}{l}
\left(V_{\mathrm{N}}^{i+1}, V_{\mathrm{T}}^{i+1}\right)=\left(0, \frac{\tilde{F}_{\mathrm{T}}^{i+1}+\mu \tilde{F}_{\mathrm{N}}^{i+1}}{\tilde{M}_{2,2}+\mu \tilde{M}_{1,2}}\right) \\
\left(R_{\mathrm{N}}^{i+1}, R_{\mathrm{T}}^{i+1}\right)=\left(\frac{\tilde{M}_{1,2} V_{\mathrm{T}}^{i+1}-\tilde{F}_{\mathrm{N}}^{i+1}}{h},-\mu R_{\mathrm{N}}^{i+1}\right)
\end{array}\right.
$$

else the particle will stick and the solution will be

$$
\left\{\begin{array}{l}
\left(V_{\mathrm{N}}^{i+1}, V_{\mathrm{T}}^{i+1}\right)=(0,0) \\
\left(R_{\mathrm{N}}^{i+1}, R_{\mathrm{T}}^{i+1}\right)=\left(-\frac{\tilde{F}_{\mathrm{T}}^{i+1}}{h},-\frac{\tilde{F}_{\mathrm{T}}^{i+1}}{h}\right),
\end{array}\right.
$$

end if

end if

end if

updating of displacement

end

$\underline{U}^{i+1}=\underline{U}^{i}+h \underline{V}^{i+1}$

Table 2 Algorithm of resolution

Proof Since a direct analytical proof of this lemma would be relatively laborious to obtain, we have analyzed the iterates of the NSCD algorithm. Let us first recall this algorithm, based on problem $\left(\mathcal{P}_{d}\right)$. A quantity with an exponent $i$ denotes the quantity at time $t_{i}$ (Table 2). $\underline{\tilde{F}}^{i+1}$ results from the integration scheme (cf. [3]) and is defined by $\underline{\tilde{F}}^{i+1}=m \underline{V}^{i}-h \underline{\underline{K}} \underline{U}^{i}+h\left[\theta \underline{F}^{i+1}+(1-\theta) \underline{F}^{i}\right], \theta$ is given in $] 0,1[, \underline{V}$ is the velocity, $\underline{V}^{\text {free }}$ is the velocity corresponding to the evolution problem without contact, and $\underline{\underline{\tilde{M}}}=m \underline{\underline{I}}+h^{2} \theta \underline{\underline{K}}$.

Let us assume that there exists a contact time $\hat{t}>0$ such that $\dot{U}_{\mathrm{T}}(\hat{t})=0$. Let $i$ be the step such that $\hat{t} \in\left[t^{i}, t^{i+1}\right]$. We want to show that if there exists an equilibrium $\left(\underline{\tilde{U}}^{\mathrm{eq}}, \underline{\tilde{R}}^{\mathrm{eq}}\right)$ such that $R_{\mathrm{N}}(\hat{t})=\tilde{R}_{\mathrm{N}}^{\mathrm{eq}}$, then $\forall j>i, \dot{U}_{\mathrm{T}}^{j} \equiv 0$, which means that the following relations (30) and (31) hold simultaneously for any $j>i$ :

$$
\begin{aligned}
& \tilde{F}_{\mathrm{T}}^{j}-\mu \tilde{F}_{\mathrm{N}}^{j}<0, \\
& \tilde{F}_{\mathrm{T}}^{j}+\mu \tilde{F}_{\mathrm{N}}^{j}>0 .
\end{aligned}
$$

We assume that the particle slips in the positive direction before step $i$ and stops at step $i+1$, that is: $\tilde{F}_{\mathrm{T}}^{i+1}-\mu \tilde{F}_{\mathrm{N}}^{i+1}<0$. We check that relation (31) is satisfied at step $j=i+1$. 

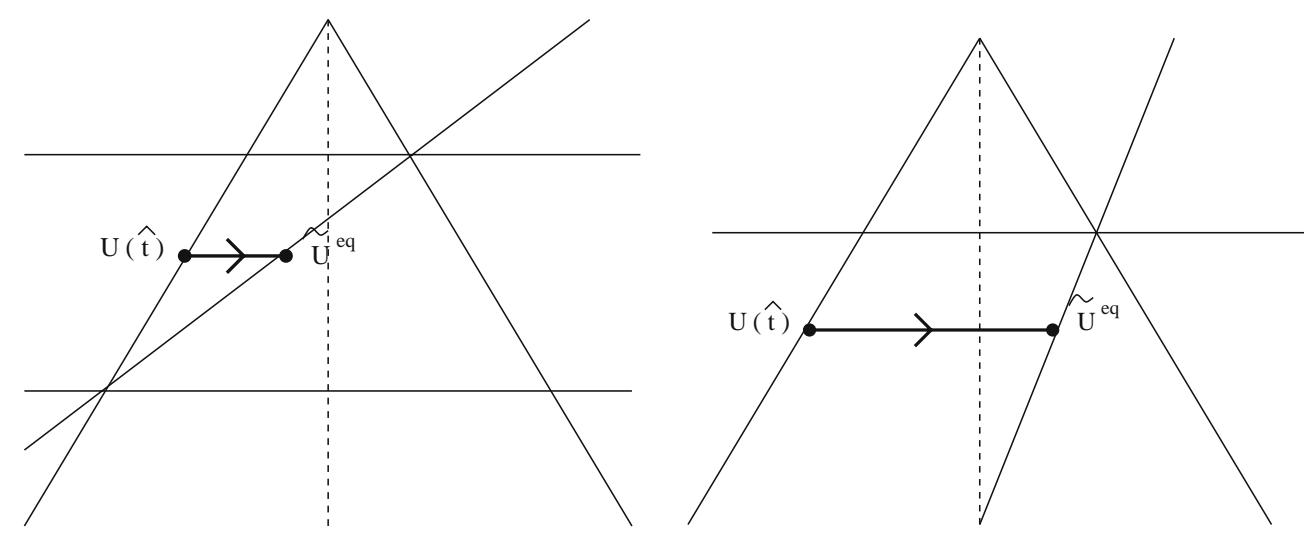

By definition,

$$
\begin{aligned}
\tilde{F}_{\mathrm{T}}^{i+1}+\mu \tilde{F}_{\mathrm{N}}^{i+1} & =m \dot{U}_{\mathrm{T}}^{i}-h\left\{\left(K_{\mathrm{T}}+\mu W\right) U_{\mathrm{T}}^{i}+\left(F_{\mathrm{T}}+\mu F_{\mathrm{N}}\right)\right\} \\
& >-h\left\{\left(K_{\mathrm{T}}+\mu W\right) U_{\mathrm{T}}(\hat{t})+\left(F_{\mathrm{T}}+\mu F_{\mathrm{N}}\right)\right\} \\
& >-h\left\{\left(K_{\mathrm{T}}+\mu W\right)\left(\frac{F_{\mathrm{N}}}{W}+\frac{\tilde{R}_{\mathrm{N}}^{\mathrm{eq}}}{W}\right)+\left(F_{\mathrm{T}}+\mu F_{\mathrm{N}}\right)\right\} \\
& >-h\left\{\frac{A}{W}+\left(K_{\mathrm{T}}+\mu W\right) \frac{\tilde{R}_{\mathrm{N}}^{\mathrm{eq}}}{W}\right\} .
\end{aligned}
$$

If $\left.\left.A=0, \tilde{R}_{\mathrm{N}}^{\mathrm{eq}} \in\right]-\infty, 0\right]$, and if $A>0$, then $\tilde{R}_{\mathrm{N}}^{\mathrm{eq}} \leq-\frac{A}{K_{\mathrm{T}}+\mu W}$. Quantity $\tilde{F}_{\mathrm{T}}^{i+1}+\mu \tilde{F}_{\mathrm{N}}^{i+1}$ is then strictly positive. Conditions (30) and (31) are therefore satisfied simultaneously. We have $\underline{\dot{U}}^{i+1} \equiv 0$, which means that $\underline{U}^{i+1}=\underline{U}^{i}$, and

$$
\tilde{F}_{\mathrm{T}}^{j}+\mu \tilde{F}_{\mathrm{N}}^{j}=-h\left\{\left(K_{\mathrm{T}}+\mu W\right) U_{\mathrm{T}}^{i}+\left(F_{\mathrm{T}}+\mu F_{\mathrm{N}}\right)\right\}>0
$$

and

$$
\tilde{F}_{\mathrm{T}}^{j}-\mu \tilde{F}_{\mathrm{N}}^{j}=-m \dot{U}_{\mathrm{T}}^{i}+\tilde{F}_{\mathrm{T}}^{i+1}-\mu \tilde{F}_{\mathrm{N}}^{i+1}<0
$$
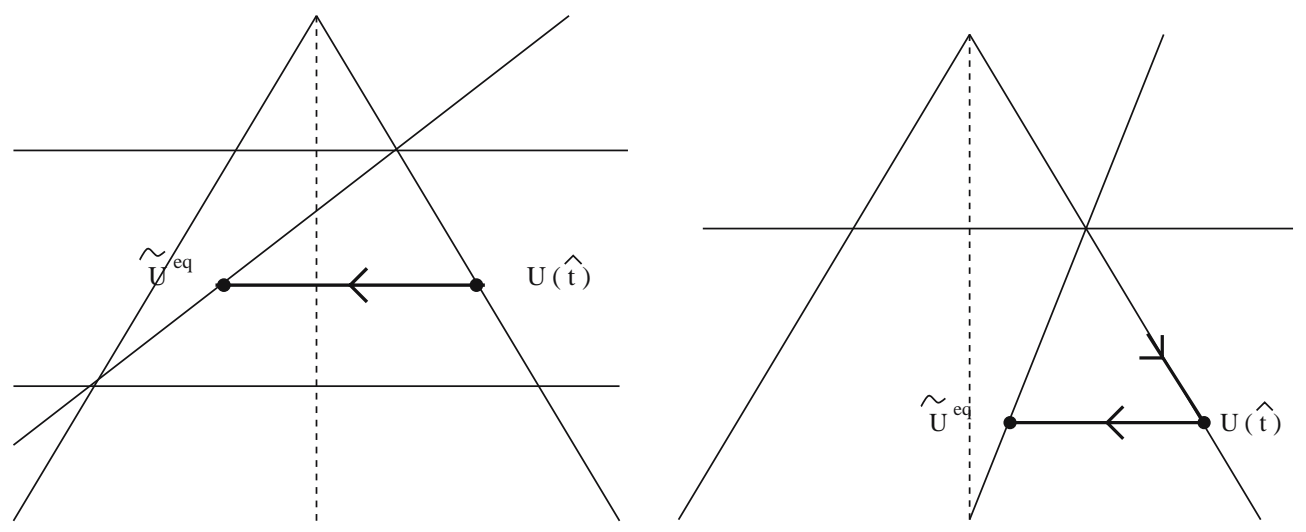

The proof is the same if the particle slips in the negative direction before step $i$. In particular, if we assume that relation (31) is satisfied at step $i+1$, then quantity $\tilde{F}_{\mathrm{T}}^{i+1}-\mu \tilde{F}_{\mathrm{N}}^{i+1}$ will be strictly negative since

$$
\tilde{F}_{\mathrm{T}}^{i+1}-\mu \tilde{F}_{\mathrm{N}}^{i+1}=m \dot{U}_{\mathrm{T}}^{i}-h\left\{\left(K_{\mathrm{T}}-\mu W\right) U_{\mathrm{T}}^{i}+\left(F_{\mathrm{T}}-\mu F_{\mathrm{N}}\right)\right\}<-h\left\{\left(K_{\mathrm{T}}-\mu W\right) U_{\mathrm{T}}^{i}+\left(F_{\mathrm{T}}-\mu F_{\mathrm{N}}\right)\right\}
$$


and:

$$
\begin{aligned}
& \text { If } \mu<\frac{K_{\mathrm{T}}}{W}, \tilde{F}_{\mathrm{T}}^{i+1}-\mu \tilde{F}_{\mathrm{N}}^{i+1}<-h\left\{\left(K_{\mathrm{T}}-\mu W\right) U_{\mathrm{T}}(\hat{t})+\left(F_{\mathrm{T}}-\mu F_{\mathrm{N}}\right)\right\} \\
& <-h\left\{\frac{A}{W}+\left(K_{\mathrm{T}}-\mu W\right) \frac{R_{\mathrm{N}}^{\mathrm{eq}}}{W}\right\}<0, \\
& \text { because } R_{\mathrm{N}}^{\mathrm{eq}} \in\left[-\frac{A}{K_{\mathrm{T}}-\mu W},-\frac{A}{K_{\mathrm{T}}+\mu W}\right] \text {, } \\
& \text { If } \mu=\frac{K_{\mathrm{T}}}{W}, \tilde{F}_{\mathrm{T}}^{i+1}-\mu \tilde{F}_{\mathrm{N}}^{i+1}<-h \frac{A}{W}<0, \\
& \text { If } \mu>\frac{K_{\mathrm{T}}}{W}, \tilde{F}_{\mathrm{T}}^{i+1}-\mu \tilde{F}_{\mathrm{N}}^{i+1}<-h\left\{\left(K_{\mathrm{T}}-\mu W\right) U_{\mathrm{T}}^{i}+\left(F_{\mathrm{T}}-\mu F_{\mathrm{N}}\right)\right\} \\
& <-h\left\{\left(K_{\mathrm{T}}-\mu W\right) \frac{F_{\mathrm{N}}}{W}+\left(F_{\mathrm{T}}-\mu F_{\mathrm{N}}\right)\right\} \\
& <-h \frac{A}{W}<0 \text {. }
\end{aligned}
$$

The following corollary is easily obtained.

Corollary 4.2 Let $A>0$.

We study the dynamical problem $(\mathcal{P})$ over $[0, \hat{T}]$ starting with a state out of equilibrium such that:

$$
\left\{\begin{array}{l}
U_{N}(0)=0, U_{\mathrm{T}}(0)=U_{\mathrm{T}}^{e q}+U_{0 T} \\
R_{\mathrm{T}}(0)= \pm \mu R_{N}(0) \neq 0 \\
\dot{U}_{N}(0)=0, \dot{U}_{\mathrm{T}}(0)=0
\end{array}\right.
$$

Then

If $\mu<K_{\mathrm{T}} / W$, there will exist nonnegative $\eta_{1}$ and $\eta_{2}, \eta_{2}>\eta_{1}$ such that for all perturbations $U_{0 T} \in$ ]$-\eta_{1}, \eta_{2}\left[\right.$, there exists $\hat{t}>0$ with $\dot{U}_{T}(\hat{t})=0, R_{N}(\hat{t})<0$.

If $\mu \geq K_{T} / W$, there will exist $\eta>0$ such that for all perturbations $\left.U_{0 T} \in\right]-\infty$, $\eta$, there exists $\hat{t}>0$ such that $\dot{U}_{T}(\hat{t})=0$ with $R_{N}(\hat{t})<0$.

Lemma 4.6 Let $A>0$ and $\mu<K_{T} / W$ and initial data be out of equilibrium such that

$$
\left\{\begin{array}{l}
U_{N}(0)=0, U_{T}(0)=U_{0 T} \\
R_{T}(0)= \pm \mu R_{N}(0) \neq 0 \\
\dot{U}_{N}(0)=0, \dot{U}_{T}(0)=0
\end{array}\right.
$$

Let $t_{i}, i=1, \ldots, n$ be a sequence of times $t_{1}<\cdots<t_{n}$ such that

$$
\left\{\begin{array}{l}
\dot{U}_{T}\left(t_{i}\right)=0 \text { with } R_{N}\left(t_{i}\right) \neq R_{N}^{e q}, \quad i=1, \ldots, n, \\
\operatorname{sgn}\left(\dot{U}_{T}^{-}\left(t_{i}\right)\right)=\operatorname{sgn}\left(\dot{U}_{T}^{-}\left(t_{i+2}\right)\right), \quad i=0, \ldots, n-2 .
\end{array}\right.
$$

Then the solution of problem $(\mathcal{P})$ with $U_{N}(t)=0$ for any $t>0$ is such that

$$
\begin{aligned}
& \text { If } \operatorname{sgn}\left(\dot{U}_{T}^{-}\left(t_{j}\right)\right)>0, j=i, i+2: U_{T}\left(t_{i}\right)>U_{T}\left(t_{i+2}\right), \quad i=1, \ldots, n ; \\
& \text { If } \operatorname{sgn}\left(\dot{U}_{T}^{-}\left(t_{j}\right)\right)<0, j=i, i+2: U_{T}\left(t_{i}\right)<U_{T}\left(t_{i+2}\right), \quad i=1, \ldots, n .
\end{aligned}
$$


Proof Here we present the proof in the case where $R_{\mathrm{T}}(0)=+\mu R_{\mathrm{N}}(0)$.

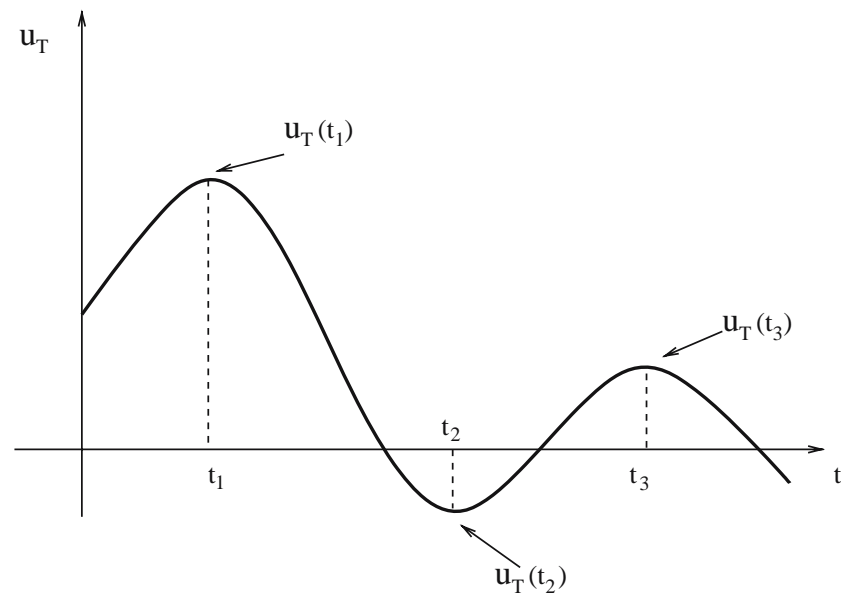

Direct analytical calculations give

$$
U_{\mathrm{T}}(t)=\left\{U_{0 \mathrm{~T}}-\frac{F_{\mathrm{T}}-\mu F_{\mathrm{N}}}{K_{\mathrm{T}}-\mu W}\right\} \cos \left(\sqrt{\frac{K_{\mathrm{T}}-\mu W}{m}} t\right)+\frac{F_{\mathrm{T}}-\mu F_{\mathrm{N}}}{K_{\mathrm{T}}-\mu W}
$$

in an interval at the right of the origin.

According to (34), there exists a time $t_{1}>0$ such that $\dot{U}_{\mathrm{T}}\left(t_{1}\right)=0$ with $U_{\mathrm{T}}\left(t_{1}\right)=-U_{0 \mathrm{~T}}+2\left(F_{\mathrm{T}}-\mu F_{\mathrm{N}}\right) /$ $\left(K_{\mathrm{T}}-\mu W\right)$.

At this time, the normal reaction is $R_{\mathrm{N}}\left(t_{1}\right)=-W U_{0 \mathrm{~T}}-\frac{2 A}{K_{\mathrm{T}}-\mu W}+F_{\mathrm{N}}$. Upon making some assumptions, $R_{\mathrm{N}}\left(t_{1}\right) \neq R_{\mathrm{N}}^{\mathrm{eq}}$. This means that

$$
U_{0 \mathrm{~T}}<-\left(2 A / W\left(K_{\mathrm{T}}-\mu W\right)\right)+\frac{A}{W\left(K_{\mathrm{T}}+\mu W\right)}+\frac{F_{\mathrm{N}}}{W}
$$

Since the tangential velocity is continuous, the particle will slip in the negative direction. The evolution is governed by a bilateral sliding process where

$$
U_{\mathrm{T}}(0)=-U_{0 \mathrm{~T}}+2 \frac{F_{\mathrm{T}}-\mu F_{\mathrm{N}}}{K_{\mathrm{T}}-\mu W}, \quad \dot{U}_{\mathrm{T}}(0)=0,
$$

so that

$$
U_{\mathrm{T}}(t)=\left\{-U_{0 \mathrm{~T}}+2 \frac{F_{\mathrm{T}}-\mu F_{\mathrm{N}}}{K_{\mathrm{T}}-\mu W}-\frac{F_{\mathrm{T}}+\mu F_{\mathrm{N}}}{K_{\mathrm{T}}+\mu W}\right\} \cos \left(\sqrt{\frac{K_{\mathrm{T}}+\mu W}{m}} t\right)+\frac{F_{\mathrm{T}}+\mu F_{\mathrm{N}}}{K_{\mathrm{T}}+\mu W}
$$

This value of $U_{\mathrm{T}}(t)$ guarantees the existence of a time $t_{2}>t_{1}$ such that $\dot{U}_{\mathrm{T}}\left(t_{2}\right)=0$. At this time, the tangential displacement is $U_{\mathrm{T}}\left(t_{2}\right)=U_{0 \mathrm{~T}}-2\left(F_{\mathrm{T}}-\mu F_{\mathrm{N}} / K_{\mathrm{T}}-\mu W\right)+2\left(F_{\mathrm{T}}+\mu F_{\mathrm{N}} / K_{\mathrm{T}}+\mu W\right)$. But the reaction is $R_{\mathrm{N}}\left(t_{2}\right) \neq R_{\mathrm{N}}^{\mathrm{eq}}$. Consequently, the particle will slip in the positive direction. The evolution is then given again by a bilateral sliding process with an initial displacement $U_{\mathrm{T}}(0)=U_{0 \mathrm{~T}}-2\left(F_{\mathrm{T}}-\mu F_{\mathrm{N}} / K_{\mathrm{T}}-\mu W\right)+$ $2\left(F_{\mathrm{T}}+\mu F_{\mathrm{N}} / K_{\mathrm{T}}+\mu W\right)$. Again there exists a time $t_{3}>t_{2}$ such that $\dot{U}_{\mathrm{T}}\left(t_{3}\right)=0$ with $U_{\mathrm{T}}\left(t_{3}\right)=-U_{0 \mathrm{~T}}+$ $4\left(F_{\mathrm{T}}-\mu F_{\mathrm{N}} / K_{\mathrm{T}}-\mu W\right)-2\left(F_{\mathrm{T}}+\mu F_{\mathrm{N}} / K_{\mathrm{T}}+\mu W\right)$. As $A>0$ and $\mu<K_{\mathrm{T}} / W$, we obviously get $U_{\mathrm{T}}\left(t_{1}\right)>$ $U_{\mathrm{T}}\left(t_{3}\right)$.

These calculations can be straightforwardly extended to any times $t_{i}, i=3, \cdots, n$. 


\section{Stability results}

We now study the stability of all the equilibrium states determined in Sect. 3. This amounts to studying the time evolution of the distance between an equilibrium and the solution of a Cauchy problem where the initial conditions are in a given neighborhood of the equilibrium. If there exists a perturbation such that the dynamics diverges from the equilibrium in a finite time or asymptotically in time, the equilibrium will be unstable. On the other hand, if no perturbation leading to a divergence exists, the equilibrium will be Lyapunov or asymptotically stable.

\section{Theorem 5.1 Any equilibrium state without contact is stable.}

Without contact the particle is not subjected to any unilateral constraints. This stability result will therefore be nothing other than the classical result of the solutions of ordinary differential equations.

Theorem 5.2 The equilibrium in grazing contact characterized by $A=0$ and $\mu<K_{T} / W$ is asymptotically stable.

Proof Although it is relatively long, the proof of this result can be obtained completely analytically. It consists of four steps.

\section{Step 1 Preliminary}

According to Comment 4.1, we only consider perturbations induced by a velocity here. Moreover, based on Lemma 4.2, the evolution is identical whether the perturbation is induced by a positive tangential velocity or by a negative normal velocity. We first show below that the study of the perturbation induced by a negative tangential velocity includes the study of the perturbation by a positive tangential velocity. Consequently, in order to prove Theorem 5.2, we will have to establish the following implication:

$$
\left.\left.\exists \eta>0, V_{0 \mathrm{~T}} \in\right]-\eta, 0\right] \Longrightarrow\left\{\begin{array}{l}
\lim _{t \rightarrow+\infty}\left|U_{\mathrm{N}}(t)\right|=0 \\
\lim _{t \rightarrow+\infty}\left|U_{\mathrm{T}}(t)-U_{\mathrm{T}}^{\mathrm{eq}}\right|=0
\end{array} .\right.
$$

We will in fact establish the stronger result:

$$
\forall V_{0 \mathrm{~T}}<0 \Longrightarrow\left\{\begin{array}{l}
\lim _{t \rightarrow+\infty}\left|U_{\mathrm{N}}(t)\right|=0 \\
\lim _{t \rightarrow+\infty}\left|U_{\mathrm{T}}(t)-U_{\mathrm{T}}^{\mathrm{eq}}\right|=0
\end{array}\right.
$$

We establish that after a negative perturbation $V_{0 \mathrm{~T}}$, there exists a time $t_{1}^{\text {vertex }}$ with $\dot{U}_{\mathrm{T}}\left(t_{1}^{\mathrm{vertex}}\right)>0$. Thus, after $t_{1}^{\text {vertex }}$, the procedure used is the same as that used to determine the evolution after the equilibrium has been perturbed by a positive tangential velocity. After a perturbation induced by a negative tangential velocity, the evolution is described by problem (20) giving the bilateral sliding with the following initial data:

$$
U_{\mathrm{T}}(0)=\frac{F_{\mathrm{N}}}{W}, \quad \dot{U}_{\mathrm{T}}(0)=V_{0_{\mathrm{T}}}<0,
$$

the solution of which is

$$
U_{\mathrm{T}}(t)=V_{0 \mathrm{~T}} \sqrt{\frac{m}{K_{\mathrm{T}}+\mu W}} \sin \left(\sqrt{\frac{K_{\mathrm{T}}+\mu W}{m}} t\right)+\frac{F_{\mathrm{T}}+\mu F_{\mathrm{N}}}{K_{\mathrm{T}}+\mu W} .
$$

Let $\hat{t}_{1}$ be the time at which $\dot{\hat{U}}_{\mathrm{T}}\left(\hat{t}_{1}\right)=0$. At this time, there is no equilibrium. The negative slip is then followed by a positive slip. During this second phase, the evolution is again described by problem (20), but with the following initial data:

$$
U_{\mathrm{T}}(0)=V_{0 \mathrm{~T}} \sqrt{\frac{m}{K_{\mathrm{T}}+\mu W}}+\frac{F_{\mathrm{T}}+\mu F_{\mathrm{N}}}{K_{\mathrm{T}}+\mu W}, \quad \dot{U}_{\mathrm{T}}(0)=0,
$$

that is,

$$
U_{\mathrm{T}}(t)=V_{0 \mathrm{~T}} \sqrt{\frac{m}{K_{\mathrm{T}}+\mu W}} \cos \left(\sqrt{\frac{K_{\mathrm{T}}-\mu W}{m}} t\right)+\frac{F_{\mathrm{T}}-\mu F_{\mathrm{N}}}{K_{\mathrm{T}}-\mu W} .
$$


Therefore there exists a time $t_{1}^{\text {vertex }}$ such that the particle reaches the vertex of the cone, which satisfies $U_{\mathrm{T}}\left(t_{1}^{\text {vertex }}\right)=F_{\mathrm{N}} / W$.

Since $A=K_{\mathrm{T}} F_{\mathrm{N}}-W F_{\mathrm{T}}=0$, this time is given by

$$
t_{1}^{\mathrm{vertex}}=\sqrt{\frac{m}{K_{\mathrm{T}}-\mu W}} \frac{\pi}{2}
$$

and therefore

$$
\dot{U}_{\mathrm{T}}\left(t_{1}^{\text {vertex }}\right)=-V_{0 \mathrm{~T}} \sqrt{\frac{K_{\mathrm{T}}-\mu W}{K_{\mathrm{T}}+\mu W}}>0,
$$

which is the end of the first step.

Step 2 The evolution of the particle includes a series of slip and motion without contact phases.

More specifically, let $t_{i}^{\mathrm{vertex}}$ be a time where the reaction reaches the vertex of the cone, as calculated in step 1 . The evolution is therefore such that there exists a part without contact, a time of impact $t_{i}^{\text {imp }}$, a new slip part, then a new time $t_{i+1}^{\text {vertex }}$, and again a new part without contact.

After $t_{i}^{\text {vertex }}$, relations (17) give

$$
\begin{aligned}
& U_{\mathrm{T}}\left(t_{i}^{\mathrm{imp}}\right)=\dot{U}_{\mathrm{T}}\left(t_{i}^{\mathrm{vertex}}\right) \frac{\sin \left(\alpha_{1} t_{i}^{\mathrm{imp}}\right)}{\alpha_{1}}+\frac{F_{\mathrm{N}}}{W}, \text { with } \sin \left(\alpha_{1} t_{i}^{\mathrm{imp}}\right)<0, \\
& \dot{U}_{\mathrm{T}}\left(t_{i}^{\mathrm{imp}}\right)=\dot{U}_{\mathrm{T}}\left(t_{i}^{\mathrm{vertex}}\right) \cos \left(\alpha_{1} t_{i}^{\mathrm{imp}}\right) .
\end{aligned}
$$

The evolution of the particle depends on the sign of the tangential velocity at impact time.

If $\dot{U}_{\mathrm{T}}\left(t_{i}{ }_{i}\right)>0$, then the slip will be in the positive direction. During this phase, the dynamics is the solution of a problem similar to (20) with

$$
U_{\mathrm{T}}(0)=U_{\mathrm{T}}\left(t_{i}^{\mathrm{imp}}\right), \quad \dot{U}_{\mathrm{T}}(0)=\dot{U}_{\mathrm{T}}\left(t_{i}^{\mathrm{imp}}\right)
$$

which we write

$$
U_{\mathrm{T}}(0)=\frac{F_{\mathrm{N}}}{W}+\beta, \quad \beta<0, \quad \dot{U}_{\mathrm{T}}(0)=0
$$

where $\beta$ is such that the forward dynamics is the same as that obtained with (38), up to a shift in time. The solution is

$$
U_{\mathrm{T}}(t)=\beta \cos \left(\sqrt{\frac{K_{\mathrm{T}}-\mu W}{m}} t\right)+\frac{F_{\mathrm{T}}-\mu F_{\mathrm{N}}}{K_{\mathrm{T}}-\mu W}
$$

and the particle reaches the vertex of the cone at time $t_{i+1}^{\mathrm{vertex}}$ such that

$$
U_{\mathrm{T}}\left(t_{i+1}^{\text {vertex }}\right)=\frac{F_{\mathrm{N}}}{W}
$$

Consequently, $\left.t_{i+1}^{\text {vertex }}=\sqrt{m /\left(K_{\mathrm{T}}-\mu W\right.}\right)(\pi / 2)$ and $\dot{U}_{\mathrm{T}}\left(t_{i+1}^{\text {vertex }}\right)=-\beta \sqrt{\left(K_{\mathrm{T}}-\mu W\right) / m}>0$.

If the velocity is positive at impact time, the particle reaches the vertex of the cone with a strictly positive velocity.

If $\dot{U}_{\mathrm{T}}\left(t_{i}\right)<0$, then the slip will be negative. We conclude in the same way that the sliding leads to the vertex of the cone with a strictly positive velocity.

From Lemma 4.2 we can observe that in any case, there is a time $t_{i+1}^{i m p}$ and that the dynamics consists of a series of phases of motion without contact and slip. 
Step 3 Let $t_{i}^{\mathrm{vertex}}, t_{i+1}^{\mathrm{vertex}}$ be two consecutive times such that $U_{\mathrm{T}}\left(t_{j}^{\mathrm{vertex}}\right)=F_{\mathrm{N}} / W, j=i, i+1$. We now establish that $\dot{U}_{\mathrm{T}}\left(t_{i+1}^{\mathrm{vertex}}\right)<\dot{U}_{\mathrm{T}}\left(t_{i}^{\mathrm{vertex}}\right)$.

If $\dot{U}_{\mathrm{T}}\left(t_{i}^{\mathrm{imp}}\right)>0$, the dynamics can be described by a bilateral phase as in problem (20) with initial data (38). During this phase, the solution is given by

$$
\begin{aligned}
U_{\mathrm{T}}(t)= & \dot{U}_{\mathrm{T}}\left(t_{i}^{\mathrm{vertex}}\right) \frac{\sin \left(\alpha_{1} t_{i}^{\mathrm{imp}}\right)}{\alpha_{1}} \cos \left(\sqrt{\frac{K_{\mathrm{T}}-\mu W}{m}} t\right) \\
& +\sqrt{\frac{m}{K_{\mathrm{T}}-\mu W}} \dot{U}_{\mathrm{T}}\left(t_{i}^{\mathrm{vertex}}\right) \cos \left(\alpha_{1} t_{i}^{\mathrm{imp}}\right) \sin \left(\sqrt{\frac{K_{\mathrm{T}}-\mu W}{m}} t\right)+\frac{F_{\mathrm{T}}-\mu F_{\mathrm{N}}}{K_{\mathrm{T}}-\mu W} .
\end{aligned}
$$

According to step 2, there exists a time $t_{i+1}^{\text {vertex }}$ where the velocity is

$$
\begin{aligned}
\dot{U}_{\mathrm{T}}\left(t_{i+1}^{\mathrm{vertex}}\right)=\dot{U}_{\mathrm{T}}\left(t_{i}^{\mathrm{vertex}}\right)\{ & -\sqrt{\frac{K_{\mathrm{T}}-\mu W}{m}} \frac{\sin \left(\alpha_{1} t_{i}^{\mathrm{imp}}\right)}{\alpha_{1}} \sin \left(\sqrt{\frac{K_{\mathrm{T}}-\mu W}{m}} t_{i+1}^{\text {vertex }}\right) \\
& \left.+\cos \left(\alpha_{1} t_{i}^{\mathrm{imp}}\right) \cos \left(\sqrt{\frac{K_{\mathrm{T}}-\mu W}{m}} t_{i+1}^{\text {vertex }}\right)\right\} .
\end{aligned}
$$

But since $\sqrt{\frac{K_{\mathrm{T}}-\mu W}{m}}<\alpha_{1}$ as soon as $W \neq 0$ and $\sin \left(\alpha_{1} t_{i}^{\mathrm{imp}}\right)<0$, then

$$
\begin{aligned}
\dot{U}_{\mathrm{T}}\left(t_{i+1}^{\text {vertex }}\right)< & \dot{U}_{\mathrm{T}}\left(t_{i}^{\text {vertex }}\right)\left\{-\sin \left(\alpha_{1} t_{i}^{\text {imp }}\right) \sin \left(\sqrt{\frac{K_{\mathrm{T}}-\mu W}{m}} t_{i+1}^{\text {vertex }}\right)\right. \\
& \left.+\cos \left(\alpha_{1} t_{i}^{\text {imp }}\right) \cos \left(\sqrt{\frac{K_{\mathrm{T}}-\mu W}{m}} t_{i+1}^{\text {vertex }}\right)\right\}, \\
< & \dot{U}_{\mathrm{T}}\left(t_{i}^{\text {vertex }}\right) \cos \left(\alpha_{1} t_{i}^{\text {imp }}+\sqrt{\frac{K_{\mathrm{T}}-\mu W}{m}} t_{i+1}^{\text {vertex }}\right), \\
< & \dot{U}_{\mathrm{T}}\left(t_{i}^{\text {vertex }}\right) .
\end{aligned}
$$

If $\dot{U}_{\mathrm{T}}\left(t_{i}^{\mathrm{imp}}\right)<0$, then the initial data are

$$
U_{\mathrm{T}}(0)=\frac{F_{\mathrm{N}}}{W}+\dot{U}_{\mathrm{T}}\left(t_{i}^{\text {vertex }}\right) \frac{\sin \left(\alpha_{1} t_{i}^{\text {imp }}\right)}{\alpha_{1}}+\beta, \beta>0, \quad \dot{U}_{\mathrm{T}}(0)=0 .
$$

In the same way, it emerges that the particle reaches the vertex at time $\left.t_{i+1}^{\mathrm{vertex}}=\sqrt{m /\left(K_{\mathrm{T}}-\mu W\right.}\right)(3 \pi / 2)$ and

$$
\begin{aligned}
\dot{U}_{\mathrm{T}}\left(t_{i+1}^{\mathrm{vertex}}\right)=-\left(\dot{U}_{\mathrm{T}}\left(t_{i}^{\mathrm{vertex}}\right) \frac{\sin \left(\alpha_{1} t_{i}^{\mathrm{imp}}\right)}{\alpha_{1}}+\beta\right) \sqrt{\frac{K_{\mathrm{T}}-\mu W}{m}} & <-\dot{U}_{\mathrm{T}}\left(t_{i}^{\mathrm{vertex}}\right) \frac{\sin \left(\alpha_{1} t_{i}^{\mathrm{imp}}\right)}{\alpha_{1}} \sqrt{\frac{K_{\mathrm{T}}-\mu W}{m}} \\
& <\frac{\dot{U}_{\mathrm{T}}\left(t_{i}^{\mathrm{vertex}}\right)}{\alpha_{1}} \sqrt{\frac{K_{\mathrm{T}}-\mu W}{m}} \\
& <\dot{U}_{\mathrm{T}}\left(t_{i}^{\mathrm{vertex}}\right) .
\end{aligned}
$$


Step 4 Let $t_{i+1}^{\mathrm{vertex}}$ be a time such that $U_{\mathrm{T}}\left(t_{i+1}^{\mathrm{vertex}}\right)=F_{\mathrm{N}} / W$, where $i$ is the index of a cycle (slip, motion without contact). Then

$$
\lim _{i \rightarrow+\infty} \dot{U}_{\mathrm{T}}\left(t_{i+1}^{\mathrm{vertex}}\right)=0
$$

According to step 3, the velocity at impact time $t_{i+1}^{\text {vertex }}$ depends on the sign of the velocity at $t_{i}^{\text {vertex }}$. But expressions (42) and (43) show that for any $i$ we can find a $\gamma_{i}, 0<\gamma_{i}<1$, with

$$
\dot{U}_{\mathrm{T}}\left(t_{i+1}^{\text {vertex }}\right)<\gamma_{i} \dot{U}_{\mathrm{T}}\left(t_{i}^{\text {vertex }}\right) \text {. }
$$

Thus

$$
\dot{U}_{\mathrm{T}}\left(t_{i+1}^{\text {vertex }}\right)<\gamma^{i} \dot{U}_{\mathrm{T}}\left(t_{1}^{\text {vertex }}\right), \text { with } \quad \gamma=\sup _{i \in \mathbb{N}} \gamma_{i}<1
$$

Consequently,

$$
\lim _{i \rightarrow+\infty} \dot{U}_{\mathrm{T}}\left(t_{i+1}^{\mathrm{vertex}}\right)=0
$$

\section{Conclusion}

If we perturb the equilibrium by applying a negative tangential velocity, steps 1 and 2 show that the particle passes through a series of phases of motion without contact and slip. According to steps 3 and 4, the velocity of the particle when it reaches the vertex of the cone will tend to zero as the time tends to infinity. Then

$$
\begin{aligned}
& \lim _{t \rightarrow+\infty}\left|U_{\mathrm{T}}(t)-U_{\mathrm{T}}^{\mathrm{eq}}\right|=0, \\
& \lim _{t \rightarrow+\infty}\left|U_{\mathrm{N}}(t)\right|=0,
\end{aligned}
$$

which is the result we were looking for.

Theorem 5.3 The equilibrium state in grazing contact $\underline{U}^{\text {affl }}=\left(0, F_{\mathrm{N}} / W\right), \underline{R}^{a f f l}=0$ characterized by $A=0$ and $\mu=K_{\mathrm{T}} / W$ is Lyapunov stable.

Proof According to Comment 4.1 and Lemma 4.2, we ought to establish the following relation:

$$
\forall \varepsilon>0, \exists \eta(\varepsilon)>0,\left|V_{0 \mathrm{~T}}\right|<\eta(\varepsilon) \Longrightarrow\left|U_{\mathrm{T}}(t)-U_{\mathrm{T}}^{\mathrm{affl}}\right|<\varepsilon .
$$

Step 1 Case of a perturbation induced by a negative tangential velocity.

After a perturbation $V_{0 \mathrm{~T}}<0$, the first slip is the solution of a bilateral problem with

$$
U_{\mathrm{T}}(0)=\frac{F_{\mathrm{N}}}{W}, \quad \dot{U}_{\mathrm{T}}(0)=V_{0 \mathrm{~T}}<0 .
$$

The tangential displacement then becomes

$$
U_{\mathrm{T}}(t)=V_{0_{\mathrm{T}}} \sqrt{\frac{m}{2 K_{\mathrm{T}}}} \sin \left(\sqrt{\frac{2 K_{\mathrm{T}}}{m} t}\right)+\frac{F_{\mathrm{N}}}{W} .
$$

The velocity is equal to zero at $\hat{t}=\sqrt{\left(m / 2 K_{\mathrm{T}}\right)}(\pi / 2)$ and $R_{\mathrm{N}}(\hat{t})=W V_{0 \mathrm{~T}} \sqrt{m / 2 K_{\mathrm{T}}}$. 


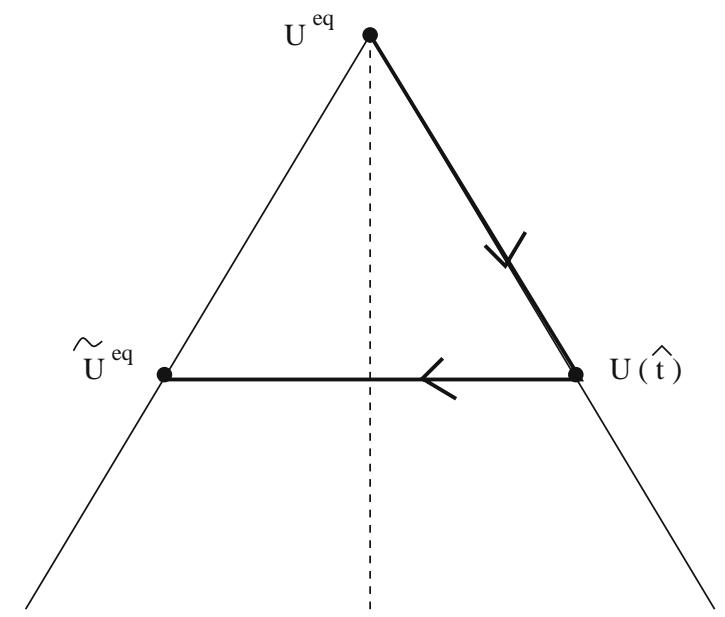

But for $A=0$ and $\mu=K_{\mathrm{T}} / W$, the range of admissible values of $R_{\mathrm{N}}$ of equilibrium states is $\mathbb{R}^{-}$. Consequently, there exists an equilibrium $\left(\underline{\tilde{U}}^{\mathrm{eq}}, \underline{\tilde{R}}^{\mathrm{eq}}\right)$ such that $\underline{\tilde{R}}^{\mathrm{eq}}=\underline{R}(\hat{t})$. Lemma 4.5 thus makes it possible to conclude that for all $t>\hat{t}$, the velocity is zero. We obtain

$$
\forall t>\hat{t},\left|U_{\mathrm{T}}^{\mathrm{affl}}-U_{\mathrm{T}}(t)\right|=\left|U_{\mathrm{T}}^{\mathrm{affl}}-U_{\mathrm{T}}(\hat{t})\right|<-V_{0_{\mathrm{T}}} \sqrt{\frac{m}{2 K_{\mathrm{T}}}} .
$$

$\forall \varepsilon>0$, relation (44) is therefore satisfied with $\eta=(\varepsilon / 2) \sqrt{2 K_{\mathrm{T}} / m}$.

Step 2 Case of a perturbation induced by a positive tangential velocity.

According to Lemma 4.2, after a perturbation $V_{0 \mathrm{~T}}>0$ there will exist an impact time $t_{1}^{\text {imp }}>0$ with a tangential velocity $\dot{U}_{\mathrm{T}}\left(t_{1}^{\mathrm{imp}}\right)$. Two situations are possible as far as the forward dynamic is concerned.

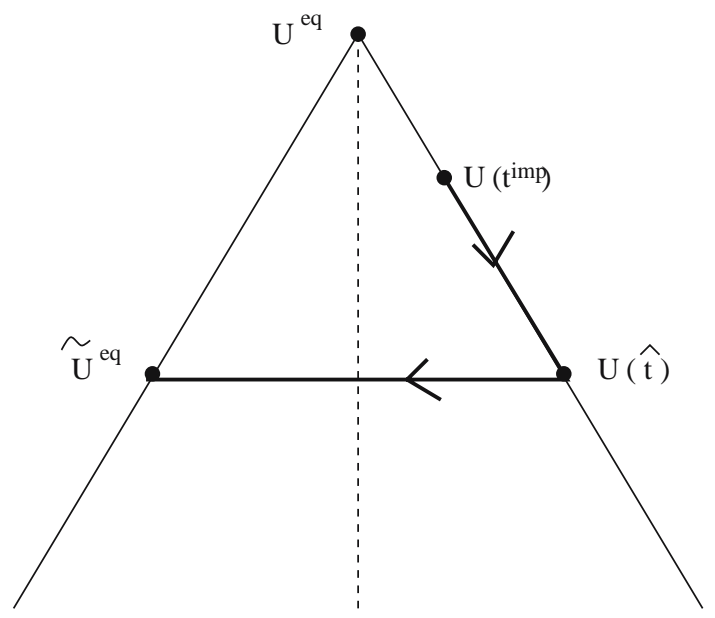

If $\dot{U}_{\mathrm{T}}\left(t_{1}^{\text {imp }}\right)<0$, a similar argument to that used in step 2 of the proof of Theorem 5.2 shows that there exists a time $\hat{t}$ such that $\dot{U}_{\mathrm{T}}(\hat{t})=0$. Since the range of admissible values of $R_{\mathrm{N}}$ is $\mathbb{R}^{-}$, there exists an equilibrium $\left(\underline{\tilde{U}}^{\mathrm{eq}}, \underline{\tilde{R}}^{\mathrm{eq}}\right)$ such that $\underline{\tilde{R}}^{\mathrm{eq}}=\underline{R}(\hat{t})$. For all $t>\hat{t}$, the velocity is therefore zero and we get

$$
\forall t>\hat{t},\left|U_{\mathrm{T}}^{\mathrm{affl}}-U_{\mathrm{T}}(t)\right|=\left|U_{\mathrm{T}}^{\mathrm{affl}}-U_{\mathrm{T}}(\hat{t})\right|<V_{0 \mathrm{~T}}\left(\frac{1}{\alpha_{1}}+\sqrt{\frac{m}{2 K_{\mathrm{T}}}}\right) .
$$


We end up in the same way as previously with $\eta=(\varepsilon / 2)\left(\left(\alpha_{1} \sqrt{2 K_{\mathrm{T}}}\right) /\left(\sqrt{2 K_{\mathrm{T}}}+\alpha_{1} \sqrt{m}\right)\right)$.

If $\dot{U}_{\mathrm{T}}\left(t_{1}^{\mathrm{imp}}\right)>0$, the proof will need a little bit more care. The particle will slip in the positive direction with a constant velocity $\dot{U}_{\mathrm{T}}\left(t_{1}^{\mathrm{imp}}\right)$. Therefore there exists a time $t_{2}^{\text {vertex }}$ such that $U_{\mathrm{T}}\left(t_{2}^{\mathrm{vertex}}\right)=\frac{F_{\mathrm{N}}}{W}$ with $\dot{U}_{\mathrm{T}}\left(t_{2}^{\text {vertex }}\right)=\dot{U}_{\mathrm{T}}\left(t_{1}^{\text {imp }}\right)>0$ followed by an impact time $t_{2}^{\text {imp }}>t_{2}^{\text {vertex }}$.

Let $t_{i}^{\text {imp }}$ and $t_{i+1}^{\text {imp }}$ be two consecutive impact times. We now show that there exists an impact time $t_{l}^{\text {imp }}$ such that $\dot{U}_{\mathrm{T}}\left(t_{l-1}^{\mathrm{imp}}\right)>0$ and $\dot{U}_{\mathrm{T}}\left(t_{l}^{\mathrm{imp}}\right)<0$. From $t_{l}^{\text {imp }}$ an argument similar to that used in step 1 makes it possible to reach the solution.

Let us assume that for all $i<l, \dot{U}_{\mathrm{T}}\left(t_{j}^{\mathrm{imp}}\right)>0, j=i, i+1$. We have

$$
\dot{U}_{\mathrm{T}}\left(t_{i+1}^{\mathrm{imp}}\right)=\dot{U}_{\mathrm{T}}\left(t_{i}^{\mathrm{imp}}\right) \cos \left(\alpha_{1} t_{i+1}^{\mathrm{imp}}\right)<\dot{U}_{\mathrm{T}}\left(t_{i}^{\mathrm{imp}}\right),
$$

which means that the tangential velocity at impact time decreases from one impact to the next. During each phase, the tangential velocity is constant and the mass will then reach the vertex of the cone with $\dot{U}_{\mathrm{T}}\left(t_{j+1}^{\mathrm{vertex}}\right)=$ $\dot{U}_{\mathrm{T}}\left(t_{j}^{\mathrm{imp}}\right)$. In addition, the tangential displacement will satisfy

$$
U_{\mathrm{T}}(t)=\dot{U}_{\mathrm{T}}\left(t_{j}^{\mathrm{imp}}\right) t+U_{\mathrm{T}}\left(t_{j}^{\mathrm{imp}}\right)
$$

Consequently, the length of the positive slip will be

$$
\Delta t_{j+1}^{\mathrm{vertex}}=-\frac{\dot{U}_{\mathrm{T}}\left(t_{j}^{\mathrm{imp}}\right)}{\dot{U}_{\mathrm{T}}\left(t_{j+1}^{\mathrm{imp}}\right)} \frac{\sin \left(\alpha_{1} t_{j+1}^{\mathrm{imp}}\right)}{\alpha_{1}} .
$$

Thus

$$
\Delta t_{i+2}^{\mathrm{vertex}}<\Delta t_{i+1}^{\mathrm{vertex}}
$$

Since the length of the evolutions without contact is identical, there exists an impact time $t_{l}^{\mathrm{imp}} \in\left[\frac{\pi}{\alpha_{1}}, 3 \pi 2 \alpha_{1}\right]+$ $2 k \pi, k \in \mathbb{N}$. Thus, $\dot{U}_{\mathrm{T}}\left(t_{l}^{\mathrm{imp}}\right)<0$.

From $t_{l}^{\text {imp }}$ onwards the particle will slip in the negative direction. This evolution is consistent with a bilateral positive sliding process given by

$$
\begin{aligned}
& m \ddot{U}_{\mathrm{T}}+\left(K_{\mathrm{T}}+\mu W\right) U_{\mathrm{T}}=F_{\mathrm{T}}+\mu F_{\mathrm{N}}, \\
& U_{\mathrm{T}}(0)=U_{\mathrm{T}}\left(t_{l}^{\mathrm{imp}}\right)=\dot{U}_{\mathrm{T}}\left(t_{l-1}^{\mathrm{imp}}\right) \frac{\sin \left(\alpha_{1} t_{l}^{\mathrm{imp}}\right)}{\alpha_{1}}+\frac{F_{\mathrm{N}}}{W}, \\
& \dot{U}_{\mathrm{T}}(0)=U_{\mathrm{T}}\left(t_{l}^{\mathrm{imp}}\right)=\dot{U}_{\mathrm{T}}\left(t_{l-1}^{\mathrm{imp}}\right) \cos \left(\alpha_{1} t_{l}^{\mathrm{imp}}\right)<0 .
\end{aligned}
$$

The solution is

$$
\begin{aligned}
U_{\mathrm{T}}(t)= & \dot{U}_{\mathrm{T}}\left(t_{l-1}^{\mathrm{imp}}\right) \frac{\sin \left(\alpha_{1} t_{l}^{\mathrm{imp}}\right)}{\alpha_{1}} \cos \left(\sqrt{\frac{2 K_{\mathrm{T}}}{m}} t\right) \\
& +\dot{U}_{\mathrm{T}}\left(t_{l-1}^{\mathrm{imp}}\right) \cos \left(\alpha_{1} t_{l}^{\mathrm{imp}}\right) \sqrt{\frac{m}{2 K_{\mathrm{T}}}} \sin \left(\sqrt{\frac{2 K_{\mathrm{T}}}{m} t}\right)+\frac{W F_{\mathrm{T}}+K_{\mathrm{T}} F_{\mathrm{N}}}{2 K_{\mathrm{T}} W} .
\end{aligned}
$$

We thus deduce the existence of the time $\hat{t}$ such that the velocity is zero. At this time, $R_{\mathrm{N}}(\hat{t})$ corresponds to an equilibrium since the range of admissible values of $R_{\mathrm{N}}$ is $\mathbb{R}^{-}$. Again, according to Lemma 4.5 , the velocity will remain equal to zero for all $t>\hat{t}$. This means that 


$$
\begin{aligned}
\forall t>\hat{t},\left|U_{\mathrm{T}}^{\mathrm{affl}}-U_{\mathrm{T}}(t)\right|< & \mid \dot{U}_{\mathrm{T}}\left(t_{l-1}^{\mathrm{imp}}\right) \frac{\sin \left(\alpha_{1} t_{l}^{\mathrm{imp}}\right)}{\alpha_{1}} \cos \left(\sqrt{\frac{2 K_{\mathrm{T}}}{m}} \hat{t}\right) \\
& +\dot{U}_{\mathrm{T}}\left(t_{l-1}^{\mathrm{imp}}\right) \cos \left(\alpha_{1} t_{l}^{\mathrm{imp}}\right) \sqrt{\frac{m}{2 K_{\mathrm{T}}}} \sin \left(\sqrt{\frac{2 K_{\mathrm{T}}}{m}} \hat{t}\right) \mid \\
< & V_{0 \mathrm{~T}} \mid \prod_{j=1}^{l-1} \frac{\sin \left(\alpha_{1} t_{j}^{\mathrm{imp}}\right)}{\alpha_{1}} \cos \left(\sqrt{\frac{2 K_{\mathrm{T}}}{m}} \hat{t}\right) \\
& +\prod_{j=1}^{l-1} \cos \left(\alpha_{1} t_{j}^{\mathrm{imp}}\right) \sqrt{\frac{m}{2 K_{\mathrm{T}}}} \sin \left(\sqrt{\frac{2 K_{\mathrm{T}}}{m}} \hat{t}\right) \mid \\
< & V_{0 T}\left(\frac{1}{\alpha_{1}}+\sqrt{\frac{m}{2 K_{\mathrm{T}}}}\right) .
\end{aligned}
$$

$\forall \varepsilon>0$, relation (44) is satisfied with $\eta=(\varepsilon / 2)\left(\alpha_{1} \sqrt{2 K_{\mathrm{T}}}\right) /\left(\sqrt{2 K_{\mathrm{T}}}+\alpha_{1} \sqrt{m}\right)$.

Theorem 5.4 All the equilibrium states in impending positive slip characterized by $A=0$ and $\mu=K_{\mathrm{T}} / W$ with a strictly negative reaction are unstable.

Proof We will now show that in the neighborhood of the equilibrium state in the impending positive slip process $\left(\underline{U}^{\mathrm{eq}}, \underline{R}^{\mathrm{eq}}\right)$, we can always find a perturbation $V_{0 \mathrm{~T}}>0$ such that the dynamics leaves this neighborhood and diverges from the equilibrium. It results from Theorem 5.3 that the solution always reaches the neighborhood of the equilibrium state under grazing contact conditions $\underline{U}^{\text {affl }}=\left(0, F_{\mathrm{N}} / W\right), \underline{R}^{\mathrm{affl}}=0$, that is,

$$
\forall \varepsilon>0 \text { and } \eta>0, \exists\left|V_{0 \mathrm{~T}}\right|<\eta \Longrightarrow\left|U_{\mathrm{T}}^{\mathrm{eq}}-U_{\mathrm{T}}(t)\right|>\varepsilon
$$

and

$$
\left|U_{\mathrm{T}}^{\mathrm{affl}}-U_{\mathrm{T}}(t)\right|<\varepsilon
$$

where $\underline{U}^{\text {affl }}$ is a finite distance from $\underline{U}^{\text {eq }}$. Then (53) gives the result.

Let an equilibrium state in an impending slip process be perturbed by an infinitesimal positive tangential velocity $V_{0 \mathrm{~T}}$. Since the velocity is constant during the positive slip, there exists a time $t_{1}^{\text {vertex }}>0$ such that the particle reaches the vertex of the cone. The assumptions made here are then the same as those made in Theorem 5.3 and as in step 2 of the proof of Theorem 5.3, i.e., the particle is no longer in contact and there exists an impact time $t_{1}^{\text {imp }}$ at which

$$
U_{\mathrm{T}}\left(t_{1}^{\mathrm{imp}}\right)=V_{0 \mathrm{~T}} \frac{\sin \left(\alpha_{1} t_{1}^{\mathrm{imp}}\right)}{\alpha_{1}}+\frac{F_{\mathrm{N}}}{W}
$$

After the impact, the evolution depends on the sign of the tangential velocity $\dot{U}_{\mathrm{T}}\left(V_{0 \mathrm{~T}}, t_{1}^{\mathrm{imp}}\left(V_{0 \mathrm{~T}}\right)\right)$.

If $\dot{U}_{\mathrm{T}}\left(t_{1}^{\mathrm{imp}}\right)<0$, then the evolution is given by (20). Consequently, there exists $\hat{t}$ such that $\dot{U}_{\mathrm{T}}(\hat{t})=0$. Since the range of values of $R_{\mathrm{N}}$ at the equilibrium is $\mathbb{R}^{-}$, there exists an equilibrium $\left(\underline{\tilde{U}}^{\mathrm{eq}}, \underline{\tilde{R}}^{\mathrm{eq}}\right)$ satisfying $\underline{R}(\hat{t})=\underline{\tilde{R}}^{\text {eq }}$. Consequently, according to Lemma $4.5, \dot{U}_{\mathrm{T}}(t) \equiv 0$ for all $t>\hat{t}$.

Since $V_{0 \mathrm{~T}}$ is infinitesimal, we obtain

$$
\begin{aligned}
\forall t>\hat{t},\left|U_{\mathrm{T}}(t)-U_{\mathrm{T}}^{\mathrm{eq}}\right| & =\left|V_{0 \mathrm{~T}} \frac{\sin \left(\alpha_{1} t_{1}^{\mathrm{imp}}\right)}{\alpha_{1}} \cos \left(\sqrt{\frac{2 K_{\mathrm{T}}}{m}} \hat{t}\right)+V_{0 \mathrm{~T}} \cos \left(\alpha_{1} t_{1}^{\mathrm{imp}}\right) \sqrt{\frac{m}{2 K_{\mathrm{T}}}} \sin \left(\sqrt{\frac{2 K_{\mathrm{T}}}{m}} \hat{t}\right)-\frac{R_{\mathrm{N}}^{\mathrm{eq}}}{W}\right|, \\
& >V_{0 \mathrm{~T}}\left|\frac{\sin \left(\alpha_{1} t_{1}^{\mathrm{imp}}\right)}{\alpha_{1}} \cos \left(\sqrt{\frac{2 K_{\mathrm{T}}}{m}} \hat{t}\right)+\cos \left(\alpha_{1} t_{1}^{\mathrm{imp}}\right) \sqrt{\frac{m}{2 K_{\mathrm{T}}}} \sin \left(\sqrt{\frac{2 K_{\mathrm{T}}}{m}} \hat{t}\right)\right| .
\end{aligned}
$$


Again as in step 2 of the proof of Theorem 5.3, we have

$$
\begin{aligned}
\forall t>\hat{t},\left|U_{\mathrm{T}}(t)-U_{\mathrm{T}}^{\mathrm{affl}}\right| & <V_{0 \mathrm{~T}}\left|\frac{\sin \left(\alpha_{1} t_{1}^{\mathrm{imp}}\right)}{\alpha_{1}} \cos \left(\sqrt{\frac{2 K_{\mathrm{T}}}{m}} \hat{t}\right)+\cos \left(\alpha_{1} t_{1}^{\mathrm{imp}}\right) \sqrt{\frac{m}{2 K_{\mathrm{T}}}} \sin \left(\sqrt{\frac{2 K_{\mathrm{T}}}{m}} \hat{t}\right)\right|, \\
& <V_{0 \mathrm{~T}}\left(\frac{1}{\alpha_{1}}+\sqrt{\frac{m}{2 K_{\mathrm{T}}}}\right) .
\end{aligned}
$$

There then exists $\eta>0$ such that the relation (52) is satisfied, for instance with $\eta=\frac{\varepsilon}{2} \frac{\alpha_{1} \sqrt{2 K_{\mathrm{T}}}}{\sqrt{2 K_{\mathrm{T}}}+\alpha_{1} \sqrt{m}}$.

The proof is obtained in the same way if $\dot{U}_{\mathrm{T}}\left(t_{1}^{\mathrm{imp}}\right)>0$.

Theorem 5.5 Let $A \geq 0$, and let $\mu$ be such that $\mu>K_{\mathrm{T}} / W$ if $A=0$ and $\mu \in \mathbb{R}$ if $A>0$. Then all the equilibrium states are Lyapunov stable.

Proof Here we have to prove the following result:

$$
\forall \varepsilon>0, \exists \eta(\varepsilon)>0,\left\|\underline{V}_{0}\right\|<\eta, \Longrightarrow\left\|\underline{U}^{\mathrm{eq}}-\underline{U}(t)\right\|<\varepsilon .
$$

It is necessary to study perturbations with $V_{0 \mathrm{~T}}<0, V_{0 \mathrm{~T}}>0$, and $V_{0 \mathrm{~N}}<0$ separately. We give the extended proof in the case $V_{0 \mathrm{~T}}<0$.

After a perturbation $V_{0 \mathrm{~T}}<0$, there exists an interval during which the motion induced is a negative slip.

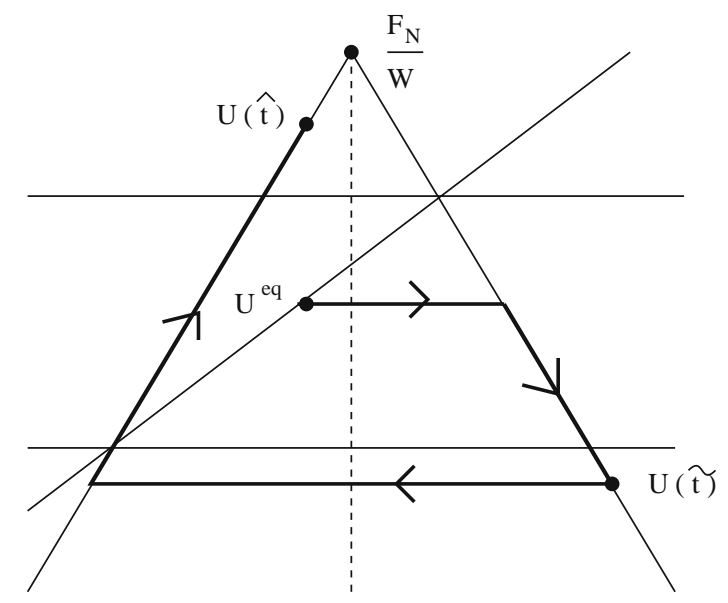

Let $A>0$ and $\mu<K_{\mathrm{T}} / W$. The negative slip phase can be followed by a positive slip phase. Consequently, the mass can reach the vertex of the cone and no longer be in contact (cf. Corollary 4.1), but the existence of this situation can be ruled out if $V_{0 \mathrm{~T}}$ is small enough, that is, $\exists \eta>0$, so that the slip stops before reaching the vertex if $\left.V_{0 \mathrm{~T}} \in\right]-\eta, 0[$.

Let us assume that the negative slip phase is followed by a positive slip phase. There exists a time $\hat{t}$ such that $\dot{U}_{\mathrm{T}}(\hat{t})=0$ with $R_{\mathrm{N}}(\hat{t})<0$.

During the negative slip phase, the evolution can be described by a bilateral problem with initial data

$$
U_{\mathrm{T}}(0)=\frac{F_{\mathrm{N}}}{W}+\frac{R_{\mathrm{N}}^{\mathrm{eq}}}{W}, \quad \dot{U}_{\mathrm{T}}(0)=V_{0 \mathrm{~T}}<0,
$$

the solution of which is

$$
\begin{aligned}
U_{\mathrm{T}}(t)= & \left(\frac{A}{W\left(K_{\mathrm{T}}+\mu W\right)}+\frac{R_{\mathrm{N}}^{\mathrm{eq}}}{W}\right) \cos \left(\sqrt{\frac{K_{\mathrm{T}}+\mu W}{m}} t\right) \\
& +V_{0 \mathrm{~T}} \sqrt{\frac{m}{K_{\mathrm{T}}+\mu W}} \sin \left(\sqrt{\frac{K_{\mathrm{T}}+\mu W}{m} t}\right)+\frac{F_{\mathrm{T}}+\mu F_{\mathrm{N}}}{K_{\mathrm{T}}+\mu W}
\end{aligned}
$$


There exists a time $\tilde{t}$ such that $\dot{U}_{\mathrm{T}}(\tilde{t})=0$, and there does not exist an equilibrium $\left(\underline{\tilde{U}}^{\mathrm{eq}}, \underline{\tilde{R}}^{\mathrm{eq}}\right)$ with $\underline{\tilde{R}}^{\mathrm{eq}}=\underline{R}(\tilde{t})$. Consequently, the evolution continues with a positive slip phase, the initial data of which are

$$
U_{\mathrm{T}}(0)=U_{\mathrm{T}}(\tilde{t})<-\frac{A}{W\left(K_{\mathrm{T}}-\mu W\right)}+\frac{F_{\mathrm{N}}}{W}, \quad \dot{U}_{\mathrm{T}}(0)=0
$$

This positive slip phase is then

$$
U_{\mathrm{T}}(t)=\left(U_{\mathrm{T}}(\tilde{t})-\frac{F_{\mathrm{T}}-\mu F_{\mathrm{N}}}{K_{\mathrm{T}}-\mu W}\right) \cos \left(\sqrt{\frac{K_{\mathrm{T}}-\mu W}{m} t}\right)+\frac{F_{\mathrm{T}}-\mu F_{\mathrm{N}}}{K_{\mathrm{T}}-\mu W},
$$

and the sliding stops before the particle reaches the vertex of the cone if

$$
\exists \hat{t}>\tilde{t} \text { such that } \dot{U}_{\mathrm{T}}(\hat{t})=0 \text { with } U_{\mathrm{T}}(\hat{t})<\frac{F_{\mathrm{N}}}{W} .
$$

Since we have

$$
\begin{aligned}
& U_{\mathrm{T}}(\hat{t})<-U_{\mathrm{T}}(\tilde{t})+2 \frac{F_{\mathrm{T}}-\mu F_{\mathrm{N}}}{K_{\mathrm{T}}-\mu W}<-\left\{\frac{A}{W\left(K_{\mathrm{T}}+\mu W\right)}+\frac{R_{\mathrm{N}}^{\mathrm{eq}}}{W}\right\} \\
& -V_{0 \mathrm{~T}} \sqrt{\frac{m}{K_{\mathrm{T}}+\mu W}}-\frac{F_{\mathrm{T}}-\mu F_{\mathrm{N}}}{K_{\mathrm{T}}-\mu W}+2 \frac{F_{\mathrm{T}}-\mu F_{\mathrm{N}}}{K_{\mathrm{T}}-\mu W},
\end{aligned}
$$

condition (58) is satisfied if

$$
V_{0 \mathrm{~T}}>\sqrt{\frac{K_{\mathrm{T}}+\mu W}{m}}\left\{-\frac{2 A}{W\left(K_{\mathrm{T}}-\mu W\right)}+\frac{A}{W\left(K_{\mathrm{T}}+\mu W\right)}\right\},
$$

which explicitly gives the value of $\eta$.

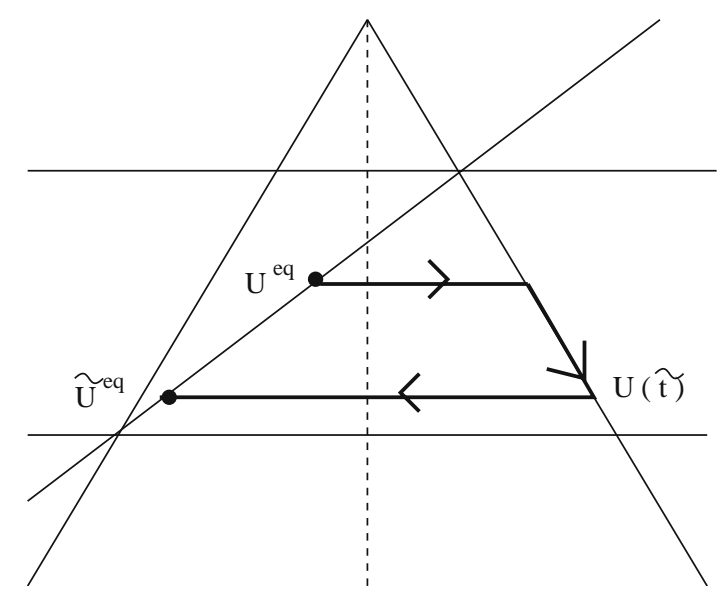

Now, let $\left(\underline{U}^{\mathrm{eq}}, \underline{R}^{\mathrm{eq}}\right)$ be an equilibrium and $V_{0 \mathrm{~T}}$ an infinitesimal negative perturbation of this equilibrium. For $A>0$ and $\mu<K_{\mathrm{T}} / W$, we assume $R_{\mathrm{N}}^{\mathrm{eq}} \neq-A /\left(K_{\mathrm{T}}-\mu W\right)$. After this perturbation, the mass slips in the negative direction. According to Lemma 4.4, there exists a time $\hat{t}>0$ such that $\underline{\dot{U}}(\hat{t})=0$, where $U_{\mathrm{T}}(\hat{t})$ is the solution of a problem similar to (47). Since the perturbation is infinitesimal, there exists an equilibrium $\left(\underline{U}^{\mathrm{eq}}, \underline{R}^{\mathrm{eq}}\right)$ at time $\hat{t}$ such that $R_{\mathrm{N}}(\hat{t})=\tilde{R}_{\mathrm{N}}^{\mathrm{eq}}$. 
We obtain

$$
\begin{aligned}
\forall t>\hat{t}, \quad & \left|U_{\mathrm{T}}^{\mathrm{eq}}-U_{\mathrm{T}}(t)\right|=\left|U_{\mathrm{T}}^{\mathrm{eq}}-U_{\mathrm{T}}(\hat{t})\right|=U_{\mathrm{T}}^{\mathrm{eq}}-U_{\mathrm{T}}(\hat{t}) \\
= & \frac{F_{\mathrm{N}}}{W}+\frac{R_{\mathrm{N}}^{\mathrm{eq}}}{W}-\left(\frac{A}{W\left(K_{\mathrm{T}}+\mu W\right)}+\frac{R_{\mathrm{N}}^{\mathrm{eq}}}{W}\right) \cos \left(\sqrt{\frac{K_{\mathrm{T}}+\mu W}{m}} \hat{t}\right) \\
& -V_{0 \mathrm{~T}} \sqrt{\frac{m}{K_{\mathrm{T}}+\mu W}} \sin \left(\sqrt{\frac{K_{\mathrm{T}}+\mu W}{m}} \hat{t}\right)-\frac{F_{\mathrm{T}}+\mu F_{\mathrm{N}}}{K_{\mathrm{T}}+\mu W}, \\
= & \frac{A}{W\left(K_{\mathrm{T}}+\mu W\right)}+\frac{R_{\mathrm{N}}^{\mathrm{eq}}}{W}-\left(\frac{W\left(K_{\mathrm{T}}+\mu W\right)}{W}\right) \cos \left(\sqrt{\frac{K_{\mathrm{T}}+\mu W}{m}} \hat{t}\right) \\
& -V_{0 \mathrm{~T}} \sqrt{\frac{m}{K_{\mathrm{T}}+\mu W}} \sin \left(\sqrt{\frac{K_{\mathrm{T}}+\mu W}{m}} \hat{t}\right), \\
< & -V_{0 \mathrm{~T}} \sqrt{\frac{m}{K_{\mathrm{T}}+\mu W}} .
\end{aligned}
$$

$\forall \varepsilon>0$, relation (56) is then satisfied with $\eta=(\varepsilon / 2) \sqrt{\left(K_{\mathrm{T}}+\mu W\right) / m}$ for $V_{0 \mathrm{~T}}<0$.

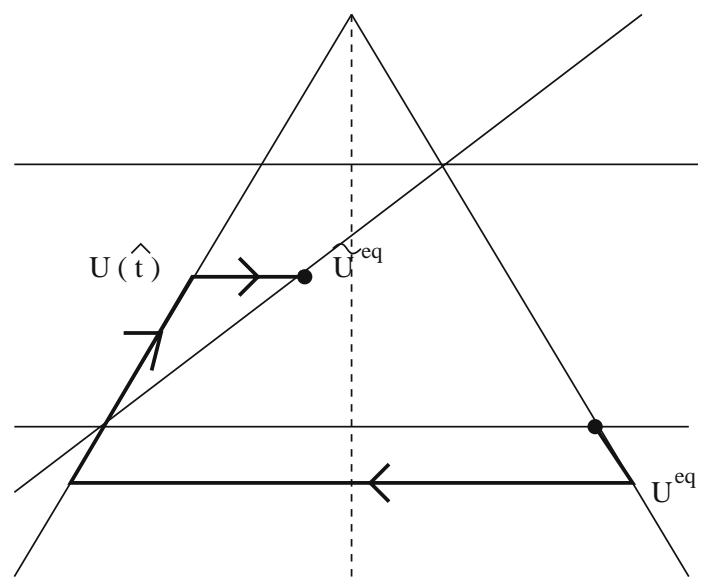

We now assume that $R_{\mathrm{N}}^{\mathrm{eq}}=-\frac{A}{K_{\mathrm{T}}-\mu W}$ for $A>0$ and $\mu<K_{\mathrm{T}} / W$. According to Lemma 4.4, there exists a time $\tilde{t}>0$ such that $\underline{\dot{U}}(\tilde{t})=0$, where $U_{\mathrm{T}}(\tilde{t})$ is given by a problem similar to (47) and $R_{\mathrm{N}}(\tilde{t})<$ $-A /\left(K_{\mathrm{T}}-\mu W\right)$. At this time $\tilde{\tilde{t}}$, the assumptions adopted in Corollary 4.2 are satisfied. The particle slips in the positive direction and there exists a time $\hat{t}>\tilde{t}$ such that $\dot{U}_{\mathrm{T}}(\hat{t})=0$. Since the perturbation $V_{0 \mathrm{~T}}$ is infinitesimal, there exists $\left(\underline{\tilde{U}}^{\mathrm{eq}}, \underline{\tilde{R}}^{\mathrm{eq}}\right)$ such that $R_{\mathrm{N}}(\hat{t})=\tilde{R}_{\mathrm{N}}^{\mathrm{eq}}$.

Indeed,

$$
R_{\mathrm{N}}(\hat{t})=W U_{\mathrm{T}}(\hat{t})-F_{\mathrm{N}}=-W U_{\mathrm{T}}(\tilde{t})+2 W \frac{\left(F_{\mathrm{N}}-\mu W\right)}{K_{\mathrm{T}}-\mu W}-F_{\mathrm{N}}
$$

However,

$$
U_{\mathrm{T}}(\tilde{t})<\frac{F_{\mathrm{N}}}{W}-\frac{A}{W\left(K_{\mathrm{T}}-\mu W\right)},
$$

and then

$$
R_{\mathrm{N}}(\hat{t})>-\frac{A}{K_{\mathrm{T}}-\mu W}
$$


Since $V_{0 \mathrm{~T}}$ is infinitesimal, we have $R_{\mathrm{N}}(\hat{t})<-A /\left(K_{\mathrm{T}}+\mu W\right)$. The range of admissible values of $R_{\mathrm{N}}$ here is $\left[-\left(\frac{A}{K_{\mathrm{T}}-\mu W}\right),-\left(\frac{A}{K_{\mathrm{T}}+\mu W}\right)\right]$. According to Lemma 4.5 , we obtain $\underline{\dot{U}}(t) \equiv 0$ for all $t>\hat{t}$. Consequently, $\forall t>\hat{t}$ :

$$
\begin{aligned}
& \left|U_{\mathrm{T}}^{\mathrm{eq}}-U_{\mathrm{T}}(t)\right|=\left|U_{\mathrm{T}}^{\mathrm{eq}}-U_{\mathrm{T}}(\hat{t})\right|<U_{\mathrm{T}}(\hat{t})-U_{\mathrm{T}}(\tilde{t}) \\
& =\left(U_{\mathrm{T}}(\tilde{t})-\frac{F_{\mathrm{T}}-\mu F_{\mathrm{N}}}{K_{\mathrm{T}}-\mu W}\right) \cos \left(\sqrt{\frac{K_{\mathrm{T}}-\mu W}{m}} \hat{t}\right) \\
& +\frac{F_{\mathrm{T}}-\mu F_{\mathrm{N}}}{K_{\mathrm{T}}-\mu W}-\left(-\frac{A}{W\left(K_{\mathrm{T}}-\mu W\right)}+\frac{A}{W\left(K_{\mathrm{T}}+\mu W\right)}\right) \cos \left(\sqrt{\frac{K_{\mathrm{T}}+\mu W}{m}} \tilde{t}\right) \\
& -V_{0 \mathrm{~T}} \sqrt{\frac{m}{K_{\mathrm{T}}+\mu W}} \sin \left(\sqrt{\frac{K_{\mathrm{T}}+\mu W}{m}} \tilde{t}\right)-\frac{F_{\mathrm{T}}+\mu F_{\mathrm{N}}}{K_{\mathrm{T}}+\mu W} \\
& <-\left(U_{\mathrm{T}}(\tilde{t})-\frac{F_{\mathrm{T}}-\mu F_{\mathrm{N}}}{K_{\mathrm{T}}-\mu W}\right)+\frac{F_{\mathrm{T}}-\mu F_{\mathrm{N}}}{K_{\mathrm{T}}-\mu W}-\left(-\frac{A}{W\left(K_{\mathrm{T}}-\mu W\right)}+\frac{A}{W\left(K_{\mathrm{T}}+\mu W\right)}\right) \\
& -V_{0 \mathrm{~T}} \sqrt{\frac{m}{K_{\mathrm{T}}+\mu W}}-\frac{F_{\mathrm{T}}+\mu F_{\mathrm{N}}}{K_{\mathrm{T}}+\mu W}, \\
& <2 \frac{F_{\mathrm{T}}-\mu F_{\mathrm{N}}}{K_{\mathrm{T}}-\mu W}-2 V_{0 \mathrm{~T}} \sqrt{\frac{m}{K_{\mathrm{T}}+\mu W}}-2 \frac{F_{\mathrm{T}}+\mu F_{\mathrm{N}}}{K_{\mathrm{T}}+\mu W}-2\left(-\frac{A}{W\left(K_{\mathrm{T}}-\mu W\right)}+\frac{A}{W\left(K_{\mathrm{T}}+\mu W\right)}\right),
\end{aligned}
$$

i.e., $\quad \forall t>\hat{t},\left|U_{\mathrm{T}}^{\mathrm{eq}}-U_{\mathrm{T}}(t)\right|<-2 V_{0 \mathrm{~T}} \sqrt{\frac{m}{K_{\mathrm{T}}+\mu W}}$.

$\forall \varepsilon>0$, relation (56) is satisfied with $\eta=\varepsilon \sqrt{\left(K_{\mathrm{T}}+\mu W\right) / m}$ for $V_{0 \mathrm{~T}}<0$.
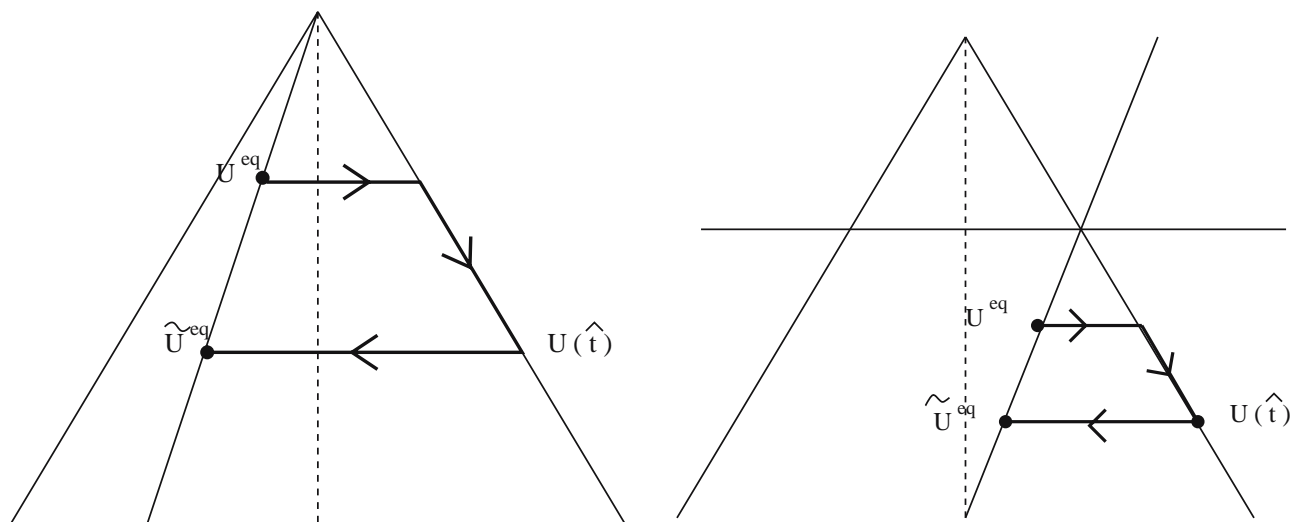

For $A=0$ and $\mu>K_{\mathrm{T}} / W$ or for $A>0$ and $\mu \geq K_{\mathrm{T}} / W$, restrictions of $V_{0 \mathrm{~T}}$ are no longer necessary, since in the case of negative slip, the reaction decreases. Consequently, since the range of the admissible values of $R_{\mathrm{N}}$ is ] $-\infty,-\frac{A}{K_{\mathrm{T}}+\mu W}$ ], there exists an equilibrium when the velocity becomes zero and the solution is reached thanks to Lemma 4.5 .

For $A>0$ and $\mu<K_{\mathrm{T}} / W$, relation (56) is satisfied for $\left.V_{0 \mathrm{~T}} \in\right]-\eta, 0[$, with $\eta$ defined at the beginning of step 1. The positive slip and negative slip phases can alternate according to $V_{0 \mathrm{~T}}$. Lemmas 4.5 and 4.6 and Corollary 4.2 prove the existence of a time $\hat{t}$ such that the velocity is zero for all $t>\hat{t}$.

The cases arising when a perturbation is induced by $V_{0 \mathrm{~T}}>0$ or by $V_{0 \mathrm{~N}}<0$ could be treated in a very similar way.

Theorem 5.6 The equilibrium state in the impending positive slip process characterized by $A<0$ and $\mu>K_{\mathrm{T}} / W$ is unstable.

This result was announced in 1990 in [5] and proved in [7] in 1995. In [7], Martins et al. obtained this result thanks to the convergence of a viscous problem.

We outline below the steps in a new proof based only on the integration of the dynamics of problem $(\mathcal{P})$. 
Proof We recall that for $A<0$ and $\mu>K_{\mathrm{T}} / W$, there exist two equilibrium solutions: one in the case of an impending positive slip process and the other in the case without contact. The impending slip solution is unstable, and the trajectory resulting from any perturbation oscillates around the solution of the problem without contact. Basically, the tools required to prove this instability result are the same as those used above. The first step consists in showing that after an initial perturbation $V_{0 \mathrm{~T}}>0$, there exists $t_{1}^{\mathrm{vertex}}$ such that $U_{\mathrm{T}}\left(t_{1}^{\text {vertex }}\right)=F_{\mathrm{N}} / W$ with $\dot{U}_{\mathrm{T}}\left(t_{1}^{\text {vertex }}\right)>0$. In the second step, we establish that the evolution of the particle includes a series of phases without contact and slip phases. Moreover, if $t_{j}^{\mathrm{vertex}}, j=i, i+1$ are two consecutive times such that the reaction reaches the vertex of the cone, we have $\dot{U}_{\mathrm{T}}\left(t_{i+1}^{\text {vertex }}\right)<\dot{U}_{\mathrm{T}}\left(t_{i}^{\text {vertex }}\right)$. The tools used here are the same as those used above, and the solution of the motion without contact always depends on the data. In the third step, it is established that, if $t_{i+1}^{\text {vertex }}$ is a time such that $U_{\mathrm{T}}\left(t_{i+1}^{\text {vertex }}\right)=F_{\mathrm{N}} / W$, then $\lim _{i \rightarrow+\infty} \dot{U}_{\mathrm{T}}\left(t_{i+1}^{\text {vertex }}\right)=0$.
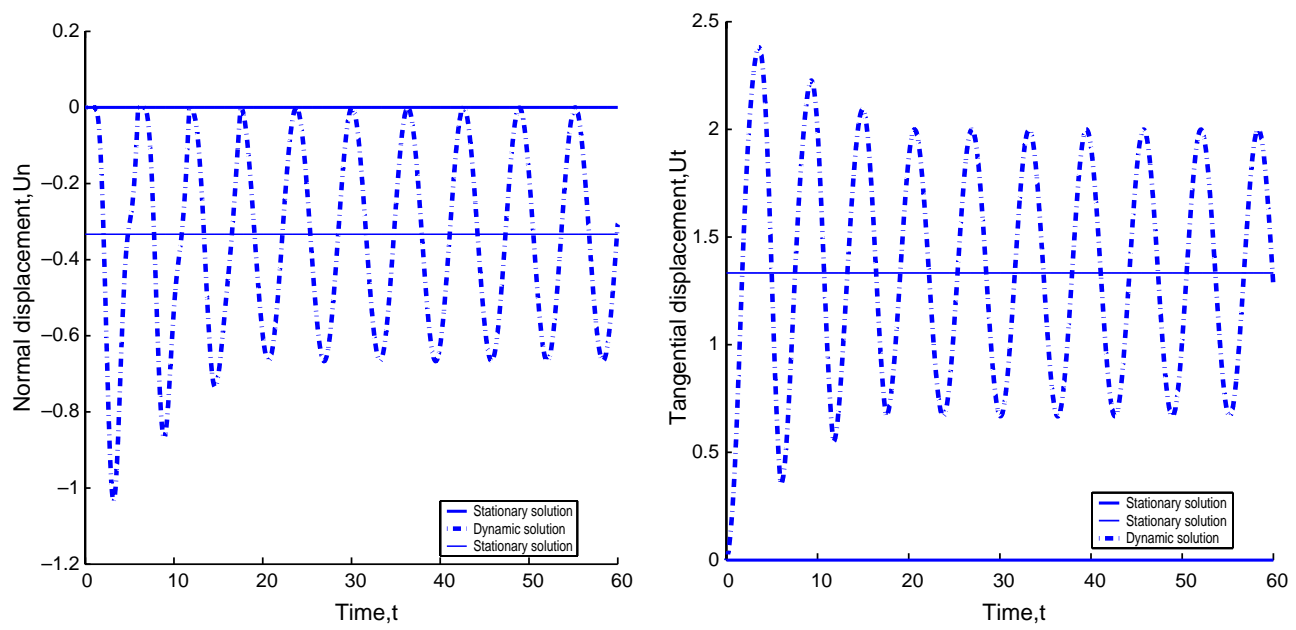

Evolution of the normal and tangential velocity after a perturbation $V_{0 \mathrm{~T}}>0$

After an infinitesimal perturbation $V_{0 \mathrm{~T}}>0$, the successive steps show that the particle passes through slip phases followed by phases without contact, followed by further slip phases, and so on. As the result of the decrease in the initial tangential velocity that occurs at each step and of the continuous dependence on the data, the dynamics converges uniformly in any compact of $[0, \hat{T}]$ toward the solution of the oscillator without contact corresponding to a perturbation of the equilibrium without contact. This proves the instability.

\section{Conclusion}

Here we studied the stability of the equilibrium states of a simple mechanical system involving unilateral contact and Coulomb friction. Since the dynamical problem is well posed for analytical external forces, we studied the stability using basic definitions of stability. The stability is studied here by directly integrating the dynamics, which follows initial data in the neighborhood of the equilibrium. The calculations are sometimes relatively long, but only quite simple tools are generally required. It is worth noting that the use of these simple tools was possible because we previously obtained theoretical results on the dynamics in the analytical case. The following three qualitative aspects of the stability properties were stressed after performing a numerical study [3]:

- The equilibrium states in impending slip processes involving a strictly negative reaction can be either stable or unstable.

- The only asymptotically stable equilibrium state is the equilibrium state in grazing contact when it is the single equilibrium state. If the equilibrium in grazing contact coexists with other equilibrium states, it is a Lyapunov stable state.

- All the equilibria in strictly stuck contact are Lyapunov stable. 
Lastly, if we look at the dynamics in the reactions space [3] (the $\left\{R_{\mathrm{T}}, R_{\mathrm{N}}\right\}$-plane), we can note that this concept of stability does not seem to perfectly suit this type of problem. Some perturbations, however small they may be, may require some large or tangential reaction jumps (from one side of the cone to the other), and small perturbations of the external force may therefore not affect the equilibrium. It therefore seems to be worth defining a new concept of stability focusing on perturbations of the equilibria induced by changes in the forces. This new definition, which was briefly mentioned in [2], will be addressed in future studies.

Let us close this work by a comment about the choice of $e=0$. As the paper deals with stability properties, it is clear that any other choice of $e \in[0,1]$ might have changed the results. But an analysis with $e \neq 0$ would not be very different from the present one. The completely analytical part of the work could be performed in exactly the same way. The other part, which uses estimates on the iterates of the NSCD algorithm of course requires $e=0$. But, as noticed at the beginning of the analysis, using only the same analytical tools as those of the other part would be possible but certainly very tedious. The present analysis of a very simple textbook case is in a sense nothing more than an example of how to deal with stability for this kind of nonsmooth system.

\section{References}

1. Ballard, P., Basseville, S.: Existence and uniqueness for dynamical unilateral contact with Coulomb friction: a model problem. Math. Model. Numer. Anal. 39(1), 57-77 (2005)

2. Basseville, S.: Dynamique et stabilité d'un système discret en présence de contact et de frottement. PhD thesis, Université d'Aix-Marseille II (2004)

3. Basseville, S., Léger, A., Pratt, E.: Investigation of the equilibrium states and of their stability for a simple model with unilateral contact and coulomb friction. Arch. Appl. Mech. 73, 409-420 (2003)

4. Jean, M.: The non-smooth contact dynamics method. Comput. Methods Appl. Mech. Eng. 177, $235-257$ (1999)

5. Klarbring, A.: Examples of non-uniqueness and non-existence of solutions to quasistatic contact problems with friction. Ing. Arch. 60, 529-541 (1990)

6. Monterio Marques M.D.P.: Differential Inclusion in Nonsmooth Mechanical Problems. Shocks and Dry Friction. Birkhäuser, Basel (1993)

7. Martins, J. A. C., Simoes, F. M. F., Gastaldi, F., Monteiro Marques, M. D. P.: Dissipative graph solutions for 2 degree-of-freedom quasistatic frictional contact problem. Int. J. Eng. Sci. 33, 1959-1986 (1995) 